\title{
Sound radiation in turbulent channel flows
}

\author{
By ZHIWEI HU ${ }^{1}$, CHRISTOPHER L. MORFEY \\ AND NEIL D. SANDHA M ${ }^{1}$
}

\author{
${ }^{1}$ Aerodynamics and Flight Mechanics Research Group, School of Engineering Sciences, \\ University of Southampton, Southampton SO17 1BJ, UK \\ ${ }^{2}$ Fluid Dynamics and Acoustics Group, ISVR, University of Southampton, \\ Southampton SO17 1BJ, UK
}

(Received 12 September 2001 and in revised form 22 August 2002)

Lighthill's acoustic analogy is formulated for turbulent channel flow with pressure as the acoustic variable, and integrated over the channel width to produce a twodimensional inhomogeneous wave equation. The equivalent sources consist of a dipole distribution related to the sum of the viscous shear stresses on the two walls, together with monopole and quadrupole distributions related to the unsteady turbulent dissipation and Reynolds stresses respectively. Using a rigid-boundary Green function, an expression is found for the power spectrum of the far-field pressure radiated per unit channel area. Direct numerical simulations (DNS) of turbulent plane Poiseuille and Couette flow have been performed in large computational domains in order to obtain good resolution of the low-wavenumber source behaviour. Analysis of the DNS databases for all sound radiation sources shows that their wavenumberfrequency spectra have non-zero limits at low wavenumber. The sound power per unit channel area radiated by the dipole distribution is proportional to Mach number squared, while the monopole and quadrupole contributions are proportional to the fourth power of Mach number. Below a particular Mach number determined by the frequency and radiation direction, the dipole radiation due to the wall shear stress dominates the far field. The quadrupole takes over at Mach numbers above about 0.1 , while the monopole is always the smallest term. The resultant acoustic field at any point in the channel consists of a statistically diffuse assembly of plane waves, with spectrum limited by damping to a value that is independent of Mach number in the low- $M$ limit.

\section{Introduction}

Turbulence is an important sound source, and the mechanism of sound production has been studied widely since Lighthill $(1952,1954)$ first explained turbulencegenerated sound by means of an acoustic analogy. He argued that the d'Alembertian $\left(\partial^{2} / \partial x_{i}^{2}-\left(1 / c_{0}^{2}\right) \partial^{2} / \partial t^{2}\right)$ of the fluid density should tend to zero far from any vorticitycontaining regions, provided the sound speed is chosen corresponding to conditions in the stationary fluid at infinity; the non-zero d'Alembertian that occurs in unbounded turbulent flow corresponds to an equivalent volume source distribution. Lighthill classified such equivalent sources as monopole, dipole and quadrupole according to their mathematical structure, and concluded that at low Mach numbers, the most important sound source in homogeneous unbounded turbulent flows is a quadrupole distribution related to fluctuating Reynolds stresses. The radiated sound intensity in this case, when scaled on a typical density, velocity and length scale of 
the flow, varies as $M^{n}$ with $n=5$. However, the other sources can be significant in certain circumstances. Morfey (1973) identified convected inhomogeneities of density or compressibility as dominant sources in turbulent flow at low Mach number, while Morfey (1976), Kambe \& Minota (1983), Kambe (1984), and Obermeier (1985) investigated the monopole sound generated by turbulent dissipation.

In most numerical studies of aerodynamic sound radiation from unbounded flows, only the Reynolds-stress quadrupoles have been considered, while viscous stresses and the entropy source term are omitted. Exceptions are the low-Re two-vortex flow models of Iafrati \& Riccardi (1996) and Mitchell, Lele \& Moin (1995), where dissipation was included but was found to have little effect. The contribution of turbulent dissipation to sound generation at higher Reynolds numbers remains controversial, and is explored further in the present paper. The geometry we have chosen (a plane infinite channel) offers two significant advantages for such a study: the acoustic solution is relatively straightforward, and the turbulent flow is statistically homogeneous in two spatial dimensions. At the same time, the presence of rigid boundaries means that viscous shear-stress dipoles appear at the channel walls (Powell 1960; Obermeier \& Möhring 1984, p. 185), and these must be included in the source description.

In a precursor to this paper, Morfey (1999) applied Lighthill's acoustic analogy to plane channel flow, and used a Green function formulation to find the plane-wave mode pressure in the waveguide formed by the rigid channel walls, for the three sources with lowest $n$ (Reynolds-stress quadrupole, dissipation monopole, and wall shear-stress dipole). The corresponding far-field intensities (per unit channel area) were then compared in terms of their Mach number dependence. Note that for each source the exponent $n$ is reduced by 1 relative to its free-field value, because of confinement by the rigid channel walls (Obermeier 1967; Ffowcs Williams \& Hawkings 1968). It was found that, provided the wavenumber-frequency spectrum of the wall shear stress is finite at zero wavenumber, the wall-shear dipole term dominates at low Mach numbers, with $n=2$; the Reynolds-stress quadrupole term and the dissipation monopole term both give $n=4$. The acoustic efficiency $\eta_{a c}$ of the various sources, defined as the ratio of the sound power radiated per unit channel area to the mean power dissipated, varies as $\eta_{a c} \sim M^{n}$. The two issues of whether the dipole term vanishes, and the relative magnitude of the quadrupole and monopole terms, are taken up in the present study.

Our approach is based on combining the Lighthill analogy with direct numerical simulation (DNS), which now can tackle turbulent flow over a range of Reynolds numbers. Detailed turbulent flow databases obtained via DNS have been successfully used in studies where experiments have difficulty in providing measurements. The simple geometry of plane channel flow has allowed investigators to run DNS up to moderate Reynolds numbers, providing mean values and higher moments of velocity and pressure, as well as kinetic energy and Reynolds stress budgets. Examples include Kim, Moin \& Moser (1987), Mansour, Kim \& Moin (1988) and Moser, Kim \& Mansour (1999) for Poiseuille flow, and Kristoffersen, Beck \& Andersson (1993) and Komminaho, Lundbladh \& Johansson (1996) for plane Couette flow. However, few simulations of plane channel flow have been carried out in a computational domain large enough to allow the streamwise and spanwise two-point correlations to drop convincingly to zero. In aeroacoustics, the low-wavenumber structure is of most concern. To obtain low-wavenumber results, the simulation domain needs to be large enough.

In the present study, simulations of Poiseuille and Couette flow, based on very large computational domains at several Reynolds numbers, are used to set up 
DNS databases for all the sound sources. Their wavenumber-frequency spectra are analysed, and their contributions to the radiated sound intensity are compared following Morfey (1999).

\section{Aeroacoustic formulation}

Throughout $\S \S 2$ to 6 , an asterisk is used to label dimensional variables; the scaled version is denoted by the same symbol without an asterisk.

\subsection{Non-dimensional formulation of the acoustic analogy}

Lighthill's acoustic analogy describes departures from a steady background state, in which the medium has uniform density and sound speed $\left(\rho_{0}^{*}, c_{0}^{*}\right)$ and is either at rest, or moves with uniform velocity. Perturbations in either pressure $\left(P^{*}-P_{0}^{*}\right)$ or density $\left(\rho^{*}-\rho_{0}^{*}\right)$ are then described by a forced wave equation, where the wave operator corresponds to freely propagating acoustic waves in the background medium.

We choose $P^{*}-P_{0}^{*}$, rather than $\rho^{*}-\rho_{0}^{*}$, as the acoustic variable in the analogy because this leads to a double time derivative, rather than a Laplacian, in the entropy source term. Conversion of one of the time derivatives to a material derivative allows this source term to be related to the dissipation function (Morfey 1976; Kambe 1984).

With all variables scaled on the reference quantities $\left(\rho_{\text {ref }}^{*}=\rho_{0}^{*}, U_{\text {ref }}^{*}, L_{\text {ref }}^{*}\right)$, the pressure version of Lighthill's acoustic analogy for an arbitrary unbounded fluid flow, acted on by external body forces, is expressed in Cartesian coordinates $x_{i}(i=1,2,3)$ by

$$
\left.\mathrm{L}(p) \equiv\left(M^{2} \frac{\partial^{2}}{\partial t^{2}}-\frac{\partial^{2}}{\partial x_{i}^{2}}\right) p=\frac{\partial^{2}}{\partial x_{i} \partial x_{j}}\left[(1+\rho) u_{i} u_{j}-\tau_{i j}\right)\right]-\frac{\partial G_{i}}{\partial x_{i}}-\frac{\partial^{2}}{\partial t^{2}}\left(\rho-M^{2} p\right) .
$$

Here $\tau_{i j}$ is the viscous stress, $G_{i}$ is the applied force per unit volume, $M$ is the scaled version of $1 / c_{0}^{*}$, and $p$ and $\rho$ are scaled pressure and density variables:

$$
M=U_{\text {ref }}^{*} / c_{0}^{*}, \quad p=\left(P^{*}-P_{0}^{*}\right) / \rho_{0}^{*} U_{\text {ref }}^{*}, \quad \rho=\left(\rho^{*}-\rho_{0}^{*}\right) / \rho_{0}^{*} .
$$

Note that the homogeneous equation $L(p)=0$ describes small-amplitude sound waves in a lossless uniform medium at rest.

\subsection{Replacement of boundaries by source and dipole layers}

The present problem differs in two important respects from that studied by Davies \& Ffowcs Williams (1968), who considered a source distribution of finite extent placed in a waveguide: in our case unsteady vorticity is present throughout the channel (i.e. volume sources extend up to the walls), and unsteady viscous stresses driven by the turbulence are present at the walls of the channel. Application of the acoustic analogy is facilitated by introducing a step function $H$ (Ffowcs Williams \& Hawkings 1969):

$$
H= \begin{cases}1 & \text { in } V \\ 0 & \text { elsewhere. }\end{cases}
$$

Here $V$ is any region of the flow field, bounded by a fixed closed surface $S$ with unit normal $\boldsymbol{b}=\left(b_{1}, b_{2}, b_{3}\right)$, pointing into $V$. In order to exclude the boundaries, we choose $S$ to lie just inside the channel walls at $x_{3}= \pm(h-\epsilon)$, with $\epsilon \downarrow 0$. Equation (1) can then be generalized to apply in all space:

$$
\mathrm{L}(p H)=\frac{\partial^{2}\left(T_{i j} H\right)}{\partial x_{i} \partial x_{j}}-\frac{\partial\left(G_{i} H+B_{i} \delta\right)}{\partial x_{i}}+\frac{\partial(Q H+B \delta)}{\partial t} .
$$


Here $\delta$ stands for the delta function whose argument $(n)$ equals the distance from $S$ measured into $V$,

$$
\delta(n)=H^{\prime}(n) \quad(n>0 \text { in } V) .
$$

In equation $(4),\left(T_{i j}, Q\right)$ are volume distributions and $\left(B_{i}, B\right)$ are surface distributions; they are defined by

$$
T_{i j}=(1+\rho) u_{i} u_{j}-\tau_{i j}, \quad Q=-\frac{\partial}{\partial t}\left(\rho-M^{2} p\right), \quad B_{i}=T_{i j} b_{j}+p b_{i}, \quad B=(1+\rho) u_{i} b_{i} .
$$

No approximations have been made up to this point. However, asymptotic ordering of the source terms requires careful treatment of the monopole term $Q$; this needs to be calculated to $O\left(M^{2}\right)$ accuracy, in order to provide an estimate of radiated pressure at low Mach numbers that is consistent with the $O(1)$ quadrupole term $T_{i j}$.

\subsection{Rearrangement of the monopole term}

We now use $\mathrm{D} / \mathrm{D} t$ to denote the material derivative and assume the fixed surface $S$ to be impermeable $\left(u_{i} b_{i}=0\right)$, so that

$$
\frac{\mathrm{D} H}{\mathrm{D} t}=0, \quad \frac{\partial H}{\partial t}=0 .
$$

By applying the identity

$$
\frac{\partial q}{\partial t} \equiv \frac{\mathrm{D} q}{D t}+q \frac{\partial u_{i}}{\partial x_{i}}-\frac{\partial\left(q u_{i}\right)}{\partial x_{i}}
$$

to the quantity $q=\left(\rho-M^{2} p\right) H=\rho_{e} H$ (say), the following equivalent expression is obtained for the volume monopole term $Q$ :

$$
Q H=R\left(1+M^{2} p\right) H-\left[\beta M^{2}+(1+\beta) M^{4} p\right] \frac{\mathrm{D}(p H)}{\mathrm{D} t}+\frac{\partial\left(\rho_{e} u_{i} H\right)}{\partial x_{i}} .
$$

Here

$$
R=\frac{\partial u_{i}}{\partial x_{i}}+M^{2}(1+\beta) \frac{\mathrm{D} p}{\mathrm{D} t}
$$

is the excess dilatation rate due to either dissipative processes or heat input, and

$$
\beta=\left(K^{*}-K_{0}^{*}\right) / K_{0}^{*}, \text { with } K^{*}=1 /\left(\rho^{*} c^{* 2}\right),
$$

is a measure of relative variations in the compressibility of the fluid. The important point is that provided the flow is adiabatic, non-reacting, and of uniform composition, then $(\rho, R, \beta) \sim M^{2}$, as shown in Appendices A and B; thus the first two terms in (9) scale as $M^{2}$ and $M^{4}$, while the third term is a dipole-order distribution that scales as $M^{2}$, in the limit of low Mach number.

The energy equation can be used to relate $R$ to the viscous dissipation function (Appendix B), leading to the following asymptotic representation of (9):

$$
Q H=\lambda M^{2} \Phi H+\frac{\partial\left(\rho_{e} u_{i} H\right)}{\partial x_{i}}+\text { (terms of order } M^{4} \text { and higher). }
$$

Here $\Phi$ is the non-dimensional dissipation function, given by

$$
\Phi=\frac{\partial u_{i}}{\partial x_{j}} \tau_{i j}, \quad \text { with } \quad \tau_{i j}=\frac{1}{R e}\left(\frac{\partial u_{i}}{\partial x_{j}}+\frac{\partial u_{j}}{\partial x_{i}}\right)+O\left(M^{2}\right) .
$$

The dimensionless coefficient $\lambda$ equals $\gamma-1$ for a perfect gas, $\gamma$ being the ratio of specific heats, but more generally it represents the Grüneisen parameter $\left(\rho^{*} / T^{*}\right) \partial T^{*} /\left.\partial \rho^{*}\right|_{s^{*}}$ 


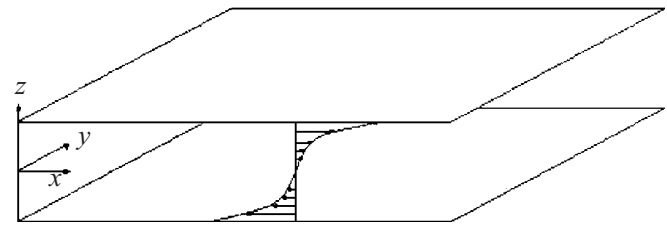

FIGURE 1. Sketch of turbulent channel (Couette) flow. The $(x, y, z)$ notation shown here is used interchangeably with $\left(x_{1}, x_{2}, x_{3}\right)$.

(Arp 1975). Other symbols $\left(T^{*}, s^{*}, R e\right)$ denote absolute temperature, specific entropy, and Reynolds number $\left(R e=\rho_{0}^{*} U_{\text {ref }}^{*} L_{\text {ref }}^{*} / \mu^{*}\right)$.

The generalized wave equation (4) is finally modified to read

$$
\mathrm{L}(p H)=\frac{\partial^{2}\left(T_{i j} H\right)}{\partial x_{i} \partial x_{j}}-\frac{\partial\left(F_{i} H+B_{i} \delta\right)}{\partial x_{i}}+\frac{\partial(Q H)}{\partial t} .
$$

Here the volume dipole distribution is given by

$$
F_{i} H=G_{i} H-\frac{\partial\left(\rho_{e} u_{i} H\right)}{\partial t}
$$

(from (4) and (12)), while $T_{i j}$ is unchanged from (6); $B$ is now zero because the fluid normal velocity vanishes on $S$, and $Q$ and $B_{i}$ are redefined as

$$
\begin{gathered}
Q=\lambda M^{2} \Phi+O\left(M^{4}\right), \\
B_{i}=-\tau_{i j} b_{j}+p b_{i} .
\end{gathered}
$$

Equations (14)-(17) describe sound radiation from weakly compressible unsteady viscous flows that are adiabatic and of uniform composition; the flow is assumed to occupy an arbitrary region with fixed impermeable boundaries, but so far no boundary geometry has been specified. In the next section, the equations are applied to the interior of a plane two-dimensional channel with rigid walls, as sketched in figure 1 , with an outgoing-wave radiation condition in directions parallel to the walls.

\subsection{Application to plane channel flow}

The cancellation implicit in the dipole and quadrupole terms of (14) has an important effect on the far-field radiation in the limit $M \ll 1$ : it introduces additional factors $\left(M, M^{2}\right)$ respectively in the radiated pressure (see $\$ 3.4$ below). To obtain the far-field pressure correct to order $M^{2}$, we use the fact that $\left(\rho, \rho_{e}\right) \sim M^{2}$ to approximate the source terms as follows:

$$
\begin{gathered}
T_{i j} \approx u_{i} u_{j}-\tau_{i j}, \quad F_{i} \approx G_{i}, \quad Q \approx \lambda M^{2} \Phi, \\
B_{i}=-\tau_{i j} b_{j}+p b_{i} .
\end{gathered}
$$

The body force $G_{i}$ has been retained, in order to drive the Poiseuille flow described in $\S 4$ without incurring a non-uniform mean pressure along the channel, but its wall-normal component is zero.

The resulting inhomogeneous wave equation (14) is most conveniently solved by using a rigid-boundary (Neumann) Green function, whose normal derivative vanishes on $S$; this eliminates the surface dipole in the normal direction, and leads to a solution in the form of acoustic channel modes (Morse \& Ingard 1986, p. 500). The channel 
modes are dispersive, making it advantageous to formulate the acoustic solution in the frequency domain. Note that for a fixed value of the scaled frequency

$$
f=\frac{f^{*} L_{\mathrm{ref}}^{*}}{U_{\mathrm{ref}}^{*}} \quad\left(f^{*}=\text { dimensional frequency }\right),
$$

the Helmholtz number based on channel half-width $h^{*}$ tends to zero in the low-Machnumber limit:

$$
H e=\frac{f^{*} h^{*}}{c_{0}^{*}}=f h M \rightarrow 0 \quad \text { as } \quad M \rightarrow 0 .
$$

When $H e$ is less than $1 / 4$, all the higher-order modes are evanescent, and only the plane-wave mode survives in the far field. This fact will be exploited in $\S 3$, where attention is focused on the plane-wave mode by integrating (14) across the channel width to yield a two-dimensional equation for the averaged instantaneous pressure $p_{\text {av }}(x, y, t)$. Here $x, y$ are streamwise and spanwise coordinates in the channel horizontal plane, as shown in figure 1.

\section{Far-field sound radiation in a plane channel}

\subsection{The role of viscous stresses}

Lighthill (1952) originally justified his lossless analogy formulation on physical grounds, by arguing that viscous attenuation of sound was a weak process at audio frequencies. A correction for such attenuation could therefore be made retrospectively. We shall follow the same approach in justifying the use (in $\S 3.2$ below) of an inviscid wave operator; for this purpose we need to show that the attenuation per wavelength is small (or at least less than 1) over the frequency range of the present simulations.

Sound waves propagating in a viscous fluid, between rigid plane parallel walls, are damped by the action of viscous shear stresses at the walls (Howe 1995; Pierce 1989, pp. 532-534). If heat conduction in the fluid is neglected, the dispersion relation for the lowest-order acoustic channel mode in the absence of mean flow may be written as

$$
\left(\frac{k^{*}}{k_{0}^{*}}\right)^{2} \approx\left(1+\frac{4}{3} \mathrm{i} \varepsilon\right)\left(1+\frac{1}{\sqrt{-\mathrm{i} \sigma}}\right) \quad(\varepsilon \ll 1, \sigma \gg 1) .
$$

Here $k^{*} / k_{0}^{*}$ is the ratio of the propagation wavenumber to the lossless acoustic wavenumber $2 \pi f^{*} / c_{0}^{*}$, and $\mathrm{i}=\sqrt{-1}$. The non-dimensional parameters $\varepsilon, \sigma$ are related to the kinematic viscosity $v *$, and are defined by

$$
\varepsilon=\frac{2 \pi f^{*} v^{*}}{c_{0}^{* 2}}=\frac{2 \pi f M^{2}}{\operatorname{Re}}, \quad \sigma=\frac{2 \pi f^{*} h^{* 2}}{v^{*}}=2 \pi f h^{2} R e ;
$$

the ratio of channel width to viscous penetration depth is $\sqrt{2 \sigma}$.

Equations (22) and (23) apply to a channel containing stationary fluid. However Howe (1995) has argued convincingly, with support from the pipe-flow experiments of Peters et al. (1993), that the no-flow attenuation coefficient given by (22) remains a valid approximation in turbulent channel flow at low Mach numbers, provided the frequency is not too low. There is a transition at low frequencies to a regime where the turbulence responds quasi-statically to the incident sound; large increases in attenuation are then possible. Quantitatively, Howe's predictions for the frequency- 
dependent attenuation coefficient $\alpha(f)$ give

$$
\begin{gathered}
\left.\alpha(f \rightarrow 0)=\alpha_{e} \simeq M_{\tau} / u_{\max } \quad \text { (quasi-static, } f<10^{-3} R e_{\tau}\right) ; \\
\left.\alpha(f \rightarrow \infty)=\alpha_{0} \simeq 0.5 M_{\tau} \sqrt{\pi f / \operatorname{Re}_{\tau}} \quad \text { (flow-independent, } f>5 \times 10^{-3} \operatorname{Re}_{\tau}\right) .
\end{gathered}
$$

All quantities in (24) and (25) are scaled using the friction velocity (indicated by the $\tau$ subscript) and the channel half-width; the maximum flow velocity in the channel is denoted by $u_{\max }$. Corresponding asymptotes for $\alpha \lambda$ (attenuation per wavelength) are

$$
\alpha \lambda(f \rightarrow 0) \simeq 1 / f u_{\max }, \quad \alpha \lambda(f \rightarrow \infty) \simeq \pi / \sqrt{2 \sigma} .
$$

The second of the expressions in (26) is always less than 1, down to the lowest frequency for which it is valid $\left(f \simeq 5 \times 10^{-3} R e_{\tau}\right)$, given that $R e_{\tau}>80$ for all the turbulent channel flow simulations presented here. It follows that at all frequencies above $f_{\min }=1 / u_{\max }$, the attenuation of the plane-wave channel mode is sufficiently weak -in the sense that $\alpha \lambda<1$ - to justify a lossless analogy formulation, provided a retrospective correction is made for attenuation (as in $\S 6.6$ below). The weakattenuation criterion sets a lower limit of $f_{\min }=0.049$ for Poiseuille flow at $R e_{\tau}=$ 360 ; the lowest frequency at this $R e_{\tau}$ for which numerical results will be presented is $f=0.39$ (figure 17). Corresponding values for $R e_{\tau}=135$ are $f_{\min }=0.057$ and $f=0.078$. (At these lowest frequencies, the channel width is respectively 42 and 11.5 times the viscous penetration depth $(\sigma=882.2,66.1)$.

Finally we note that neglect of viscous damping would lead, in a channel of infinite extent, to an infinite mean-square radiated pressure (Olbers's paradox). Nevertheless we are able to bypass the damping issue initially, by presenting results for the sound power radiated per unit area of channel. Only in $\S 6.6$, where we estimate the local mean-square pressure in the channel due to acoustic radiation, is it necessary to introduce the damping explicitly.

\subsection{Two-dimensional form of the wave equation}

The use of rigid-wall boundary conditions to solve (14) eliminates the normal surface dipoles $B_{3}$ on both walls of the channel. Integration over the channel width $\left(x_{3}=-h\right.$ to $\left.x_{3}=h\right)$ leads to a two-dimensional inhomogeneous wave equation in scaled variables,

$$
\mathrm{L}_{2}\{p(\boldsymbol{x}, t)\}=\frac{\partial^{2} t_{\alpha \beta}}{\partial x_{\alpha} \partial x_{\beta}}-\frac{\partial f_{\alpha}}{\partial x_{\alpha}}+\frac{\partial q}{\partial t}=S(\boldsymbol{x}, t) ;
$$

the two-dimensional wave operator is defined by $\mathrm{L}_{2} \equiv M^{2} \partial^{2} / \partial t^{2}-\partial^{2} / \partial x_{\alpha}^{2}$, and $x_{\alpha}$ $(\alpha=1,2)$ are the Cartesian components of the two-dimensional position vector $\boldsymbol{x}$ in the horizontal plane of the channel.

The pressure $p$ is now the average over the channel width, previously denoted by $p_{\text {av }}$ : it represents the pressure in the plane-wave mode, for the waveguide formed by the channel walls (see the final paragraph of $\S 2$ ). Propagation of the plane-wave mode is described to lowest order in $M$ by a convected wave equation, with the background medium moving at the bulk mean velocity of the channel flow. The effects of convection on the sound field are not included in the wave operator on the left of (27), on the basis that they appear as order- $M$ corrections to the estimates presented in this paper (Pagneux \& Froehlich 2001). The effects of refraction on the plane-wave mode are of higher order again in Mach number. 
The multipole distributions on the right of equation (27) are given by

$$
\begin{gathered}
\text { quadrupole: } \quad t_{\alpha \beta}=\frac{1}{2 h} \int_{-h}^{h} T_{\alpha \beta} \mathrm{d} x_{3} \quad\left(T_{\alpha \beta} \approx u_{\alpha} u_{\beta}-\tau_{\alpha \beta}\right), \\
\text { dipole: } f_{\alpha}=-\frac{1}{2 h}\left(\tau_{\alpha}^{(u)}+\tau_{\alpha}^{(l)}\right)^{\prime}=\frac{1}{2 h R e}\left\{\left(\frac{\partial u_{\alpha}}{\partial x_{3}}\right)^{(u)}-\left(\frac{\partial u_{\alpha}}{\partial x_{3}}\right)^{(l)}\right\}^{\prime}, \\
\text { monopole: } \quad q=\lambda M^{2} \Psi, \quad \text { with } \Psi=\frac{1}{2 h} \int_{-h}^{h} \Phi \mathrm{d} x_{3} .
\end{gathered}
$$

In equation (29), $\tau_{\alpha}$ denotes the viscous shear force on the wall per unit area, primes indicate that the time-average value has been subtracted out, and superscripts $(u),(l)$ refer to $x_{3}=+h,-h$, respectively. Note that when the wall shear forces per unit area, $\tau_{\alpha}= \pm \tau_{\alpha 3}$, are expressed in the wavenumber domain as $\tau_{\alpha}\left(k_{x}, k_{y}, t\right)$, the zerowavenumber component $\tau_{\alpha}(0,0, t)$ does not necessarily vanish, whereas the viscous stress components $\tau_{\alpha \beta}$ do have $\tau_{\alpha \beta}(0,0, t)=0$ for incompressible flow. Anticipating the analysis of $\S 3.4$, it can be seen that the viscous part of the quadrupole $t_{\alpha \beta}$ in (28) will not contribute to sound radiation in the present low-Mach-number approximation.

\subsection{Frequency-domain solution}

It is easier to solve (27) in the frequency domain. Fourier transformation leads to a Helmholtz equation,

$$
\left(\frac{\partial^{2}}{\partial x_{\alpha}^{2}}+k_{0}^{2}\right) p(\boldsymbol{x}, f)=-S(\boldsymbol{x}, f) ;
$$

here $f$ is the non-dimensional frequency, and $k_{0}$ is the non-dimensional acoustic wavenumber,

$$
k_{0}=2 \pi f^{*} L_{r e f}^{*} / c_{0}^{*}=2 \pi f M .
$$

The definition used for transforming any variable $v(\boldsymbol{x}, t)$ from time to frequency is

$$
v(\boldsymbol{x}, f)=\int_{-\infty}^{+\infty} v(\boldsymbol{x}, t) \mathrm{e}^{\mathrm{i} 2 \pi f t} \mathrm{~d} t .
$$

The sign convention in the exponential factor is the opposite of that used later for spatial Fourier transforms, and is chosen for convenience in interpreting wave propagation directions.

Let $g(\boldsymbol{x} \mid \boldsymbol{y})$ be the point-source solution of (31) with $S$ replaced by the twodimensional delta function $\delta(x-y)$; then the pressure field due to the source distribution $S$ is

$$
p(\boldsymbol{x}, f)=\int S(\boldsymbol{y}, f) g(\boldsymbol{x} \mid \boldsymbol{y}) \mathrm{d}^{2} \boldsymbol{y},
$$

where the integral extends over the entire two-dimensional source region. Because equation (27) is valid everywhere (the channel is of infinite extent), and has constant coefficients, the quadrupole and dipole contributions to the pressure can be expressed as

$$
p_{\text {quad }}=\frac{\partial^{2}}{\partial x_{\alpha} \partial x_{\beta}} \int g t_{\alpha \beta} \mathrm{d}^{2} \boldsymbol{y}, \quad p_{\text {dip }}=-\frac{\partial}{\partial x_{\alpha}} \int g f_{\alpha} \mathrm{d}^{2} \boldsymbol{y},
$$

provided the integrals converge (Lighthill 1978, pp. 62-63). This result allows replacement of the actual source, $S(\boldsymbol{x}, f)$, in (31) by an equivalent source distribution for 
far-field radiation, $\Gamma(\boldsymbol{x}, f)$, whose value depends on the radiation direction as specified by the unit vector $\boldsymbol{e}=\left(e_{1}, e_{2}\right)$ :

$$
\Gamma(\boldsymbol{x}, f)=\left(\mathrm{i} k_{0}\right)^{2} e_{\alpha} e_{\beta} t_{\alpha \beta}-\mathrm{i} k_{0} e_{\alpha} f_{\alpha}-\mathrm{i} 2 \pi f q .
$$

The $\left(\mathrm{i} k_{0}\right)$ factors on the right of (36) arise from the far-field Green function, as discussed below. The absence of spatial and temporal derivatives in this result, compared with the original $S(\boldsymbol{x}, t)$ expression (27), simplifies the computation of radiated sound; furthermore the inherent quadrupole and dipole cancellation is allowed for in the $k_{0}^{2}$ and $k_{0}$ factors (proportional to $M^{2}, M$ respectively).

\subsection{Far-field radiation from a finite region of turbulence}

We now consider the radiation emitted by a finite sub-region of the source (in two space dimensions and time), defined by $0<x_{1}<L_{1}, 0<x_{2}<L_{2}$, and $0<t<T$. The source distribution $S(\boldsymbol{y}, t)$ and the corresponding radiated pressure $p(\boldsymbol{x}, t)$ then both have finite Fourier transforms with respect to $t$, and the power spectral density of the radiated pressure in the channel, normalized to unit channel area, is given by

$$
s_{p}(\boldsymbol{x}, f)=\mathrm{E}\left\{\lim _{T, L_{1}, L_{2} \rightarrow \infty} \frac{1}{T L_{1} L_{2}}|p(\boldsymbol{x}, f)|^{2}\right\} .
$$

Our aim is to evaluate (37) for positions $\boldsymbol{x}$ such that $|\boldsymbol{x}| \gg\left(L_{1}, L_{2},(f M)^{-1}\right)$.

The frequency-domain far-field pressure is given by the Green function integral (34), with $S$ replaced by $\Gamma$ from (36), and with the following expression for the outgoing-wave Green function in two dimensions that describes the plane-wave mode in the channel (see $\S 2.4)$ :

$$
\begin{aligned}
g(\boldsymbol{x} \mid \boldsymbol{y}) & =\frac{1}{4} \mathrm{i} H_{0}^{(1)}\left(k_{0} r\right) \quad\left(\mathrm{e}^{-\mathrm{i} 2 \pi f t} \text { convention; } r=|\boldsymbol{x}-\boldsymbol{y}|\right) \\
& \approx \frac{1}{\left(8 \pi k_{0} r\right)^{1 / 2}} \mathrm{e}^{\mathrm{i}\left(k_{0} r+\pi / 4\right)} \quad\left(k_{0} r \gg 1\right) .
\end{aligned}
$$

Here $H_{0}^{(1)}$ is the zero-order Hankel function $J_{0}+\mathrm{i} Y_{0}$. Since in the far field

$$
\partial g(\boldsymbol{x} \mid \boldsymbol{y}) / \partial x_{\alpha} \approx e_{\alpha} \partial g / \partial r \approx \mathrm{i} k_{0} e_{\alpha} g,
$$

it can be seen that (36) follows from (35). The Green function (38) is further simplified by applying the Fraunhofer approximation,

$$
r \approx|x|-e \cdot y,
$$

to the exponential term. This gives the far-field result

$$
p(\boldsymbol{x}, f) \approx \frac{\mathrm{e}^{\mathrm{i}\left(k_{0}|\boldsymbol{x}|+\pi / 4\right)}}{\left(8 \pi k_{0}|\boldsymbol{x}|\right)^{1 / 2}} \int_{0}^{L_{2}} \int_{0}^{L_{1}} \Gamma\left(y_{1}, y_{2}, f\right) \mathrm{e}^{-\mathrm{i} k_{0}\left(e_{1} y_{1}+e_{2} y_{2}\right)} \mathrm{d} y_{1} \mathrm{~d} y_{2} .
$$

The double integral over $y_{1}$ and $y_{2}$ can be recognized as a two-dimensional spatial Fourier transform of the equivalent source distribution, $\Gamma(\boldsymbol{k}, f)$, evaluated at wavenumber $\boldsymbol{k}=k_{0} \boldsymbol{e}$.

Finally, substituting (41) in (37) gives

$$
s_{p}(\boldsymbol{x}, f) \approx \frac{1}{8 \pi k_{0}|\boldsymbol{x}|} \mathrm{E}\left\{\lim _{T, L_{1}, L_{2} \rightarrow \infty} \frac{1}{T L_{1} L_{2}}|\Gamma(\boldsymbol{k}, f)|^{2}\right\}_{\boldsymbol{k}=k_{0} e} .
$$

The expected value on the right of $(42)$, when divided by $(2 \pi)^{2}$, becomes the threedimensional power spectral density of $\Gamma(\boldsymbol{x}, t)$ in wavenumber $\left(k_{1}, k_{2}\right)$ and frequency $(f)$; 
the $(2 \pi)^{2}$ arises from the use of angular wavenumber, as is conventional in fluid mechanics and acoustics. With $S_{\Gamma}(\boldsymbol{k}, f)$ used to denote this wavenumber-frequency spectrum, an equivalent version of (42) is

$$
s_{p}(\boldsymbol{x}, f) \approx \frac{1}{4 f M|x|} S_{\Gamma}\left(k_{0} e, f\right) \quad(f M|x| \gg 1) .
$$

Note that the far-field acoustic intensity per unit channel area has a frequency spectrum equal to $M s_{p}(\boldsymbol{x}, f)$; the additional factor $M$ cancels the $1 / M$ on the right of (43).

\subsection{DNS application}

All components of the multipole distribution defined in (28) to (30) have been calculated for fully developed turbulent channel flow, using the DNS scheme described in $\S 4$. Time series of the separate source terms are stored in a database as a function of simulation wavenumber components $k_{1}$ (streamwise) and $k_{2}$ (spanwise). In a postprocessing operation, the time series for each term at each wavenumber is divided into eight non-overlapping time segments of equal length. Each time segment has its mean subtracted and a Hanning window applied, before Fourier transformation into the frequency domain with (33) to yield an estimate of the corresponding term in $\Gamma(\boldsymbol{k}, f)$. The power spectrum $S_{\Gamma}(\boldsymbol{k}, f)$ is then obtained from

$$
S_{\Gamma}(\boldsymbol{k}, f)=\frac{1}{(2 \pi)^{2}} \mathrm{E}\left\{\lim _{T, L_{1}, L_{2} \rightarrow \infty} \frac{1}{T L_{1} L_{2}}|\Gamma(\boldsymbol{k}, f)|^{2}\right\} .
$$

In equation (44), the expected value is estimated by first smoothing in frequency and then averaging over the eight time segments. Frequency smoothing is implemented as a seven-point moving average based on seven successive frequency bins; note the frequency resolution of the transforms can be read from the frequency spectra, since it equals the lowest frequency plotted. Examples of source spectra are presented in $\S 6$, for both Poiseuille and Couette flow.

\section{DNS of incompressible plane channel flow}

\subsection{Governing equations and summary of numerical method}

The governing continuity and momentum equations of incompressible turbulent flow are non-dimensionalized using a reference length $L_{\text {ref }}^{*}$ equal to the channel halfwidth $h^{*}$, and with the reference velocity, $U_{\text {ref }}^{*}$, chosen as the friction velocity $u_{\tau}^{*}$ for Poiseuille flow, and the wall velocity $u_{w}^{*}$ for Couette flow (both the upper and the lower walls move, with velocity $u_{w}^{*}$ and $-u_{w}^{*}$ respectively). The non-dimensional continuity equation and the rotation form of the momentum equation can be written as

$$
\begin{gathered}
\frac{\partial u_{j}}{\partial x_{j}}=0 \\
\frac{\partial u_{i}}{\partial t}=\epsilon_{i j k} u_{j} \omega_{k}+\delta_{1 i} \Lambda-\frac{\partial \Pi}{\partial x_{i}}+\frac{1}{R e} \frac{\partial^{2} u_{i}}{\partial x_{j} \partial x_{j}} .
\end{gathered}
$$

Here $\omega_{i}$ is the vorticity component, $\omega_{i}=\epsilon_{i j k} \partial u_{k} / \partial x_{j}$; Reynolds number is $R e=$ $U_{\text {ref }}^{*} h^{*} / v^{*}$, equal to $R e_{\tau}=u_{\tau}^{*} h^{*} / v^{*}$ for Poiseuille flow, and $R e_{w}=u_{w}^{*} h^{*} / v^{*}$ for Couette flow; $v^{*}$ is the kinematic viscosity of the fluid; $\Pi=p+u_{i} u_{i} / 2$ is the non-dimensional modified pressure; $\epsilon_{i j k}$ is the permutation tensor; and $\delta_{1 i} \Lambda$ is the driving mean pressure gradient, equivalent to a body force $G_{i}$. 
Poiseuille flow is a pressure-gradient-driven flow. Taking the Reynolds average (denoted by the overbar) of the streamwise momentum equation, we obtain

$$
\Lambda=\frac{\partial}{\partial z}\left(\overline{u^{\prime} w^{\prime}}-\bar{\tau}_{x z}\right)
$$

where $\bar{\tau}_{x z}=(1 / R e) \partial \bar{u}_{x} / \partial z$ is the mean viscous shear stress. The non-dimensional mean pressure gradient $\Lambda=1$ in plane Poiseuille flow, so the total non-dimensional shear stress equals $z$. On the other hand, plane Couette flow is driven by the movement of the walls. There is no mean pressure gradient for Couette flow, and the total shear stress is constant across the channel.

Numerical solution of equations (45), (46) follows the spectral method of Kleiser \& Schumann (1980) (see also Canuto et al. 1987, pp. 79-81), with Fourier and Chebyshev methods used for spatial discretizations, and an implicit treatment of pressure and viscous terms to avoid extremely small time steps in the near-wall region. The AdamsBashforth time advance of Kleiser \& Schumann (1980) was replaced by a third-order Runge-Kutta method for the convective terms and the whole method was parallelized (Sandham \& Howard 1998).

\subsection{Spatial derivatives}

Spectral methods are an accurate way of forming spatial derivatives, for wavenumbers below some upper limit set by the spatial resolution. Fourier spectral methods require periodic boundary conditions, while Chebyshev methods can be applied to nonperiodic directions. In the present problem, Fourier discretization is used for the channel horizontal plane, and the Chebyshev tau method is used for the wall-normal direction.

In the horizontal plane, a two-dimensional Fourier transformation from real to wave space is accomplished by a streamwise real to complex Fourier transformation, followed by a complex to complex Fourier transformation in the spanwise direction. After the streamwise transformation, only the mean and the positive Fourier modes need to be stored owing to symmetry. A real quantity $q(x, y)$ is transformed to $\tilde{q}\left(k_{x}, k_{y}\right)$ in discrete Fourier space by the successive operations:

$$
\begin{gathered}
\tilde{q}\left(k_{x_{l}}, y_{j}\right)=\frac{1}{N_{x}} \sum_{i=0}^{N_{x}-1} q\left(x_{i}, y_{j}\right) \mathrm{e}^{-\mathrm{i} 2 \pi l i / N_{x}}, \\
\tilde{q}\left(k_{x_{l}}, k_{y_{m}}\right)=\frac{1}{N_{y}} \sum_{j=0}^{N_{y}-1} \tilde{q}\left(k_{x_{l}}, y_{j}\right) \mathrm{e}^{-\mathrm{i} 2 \pi m j / N_{y}},
\end{gathered}
$$

where $x_{i}, y_{j}$ are the streamwise and spanwise coordinates of grid points with a total number of $N_{x}$ and $N_{y}$ in the two directions (both even). Uniform grids are used, so

$$
x_{i}=\frac{L_{x} i}{N_{x}}\left(0 \leqslant i \leqslant N_{x}\right), \quad y_{j}=\frac{L_{y} j}{N_{y}}\left(0 \leqslant j \leqslant N_{y}\right) .
$$

The spatial wavenumbers $k_{x_{l}}, k_{y_{m}}$ are given by

$$
k_{x_{l}}=\frac{2 \pi l}{L_{x}}\left(-N_{x} / 2 \leqslant l \leqslant N_{x} / 2\right), \quad k_{y_{m}}=\frac{2 \pi m}{L_{y}}\left(-N_{y} / 2 \leqslant m \leqslant N_{y} / 2\right),
$$

where $L_{x}$ and $L_{y}$ are the non-dimensional computational box lengths in the streamwise and spanwise directions. 
The two-dimensional backward transformation is done with a spanwise complex to complex transformation, followed by a streamwise complex to real transformation:

$$
\begin{gathered}
\tilde{q}\left(k_{x_{l}}, y_{j}\right)=\sum_{m=-N_{y} / 2}^{N_{y} / 2} \tilde{q}\left(k_{x_{l}}, k_{y_{m}}\right) \mathrm{e}^{\mathrm{i} 2 \pi m j / N_{y}}, \\
q\left(x_{i}, y_{j}\right)=\tilde{q}\left(0, y_{j}\right)+2 \sum_{l=1}^{N_{x} / 2} \tilde{q}\left(k_{x_{l}}, y_{j}\right) \mathrm{e}^{\mathrm{i} 2 \pi l i / N_{x}} .
\end{gathered}
$$

Chebyshev transformations are used for the wall-normal direction with the help of the Chebyshev polynomial $T_{n}\left(z_{k}\right)$ (Canuto et al. 1987, pp. 79-81):

$$
\begin{aligned}
& \tilde{q}(n)=\sum_{k=0}^{N_{z}-1} q\left(z_{k}\right) T_{n}\left(z_{k}\right), \\
& q\left(z_{k}\right)=\sum_{n=0}^{N_{z}-1} \tilde{q}(n) T_{n}\left(z_{k}\right) .
\end{aligned}
$$

Here $N_{z}$ is the number of wall-normal grid points, whose coordinates $z_{k}$ are a non-uniform cosine profile

$$
z_{k}=\cos \left(\frac{\pi k}{N_{z}-1}\right) \quad\left(0 \leqslant k \leqslant N_{z}-1\right) .
$$

The nonlinear convective terms are calculated by zero-padding the separate factors in wave space by $50 \%$ before transforming back to real space, where the nonlinear terms are calculated. This ' $3 / 2$ rule' de-aliasing has been applied whenever nonlinear quantities are required. Note that the process generates additional wavenumber components in the wave-space representation, but these are truncated.

\subsection{Initial condition}

The initial flow fields consist of an approximate mean turbulent flow with superimposed artificial disturbances. Statistical data are accumulated only after the initial influence has disappeared and the flow has statistically settled down. The convergence is checked by comparing the statistical data in successive time segments, making sure that they are consistent. All statistical data are averaged over the horizontal plane and time.

\section{Validation of DNS}

\subsection{Plane Poiseuille flow}

The computational domain used for Poiseuille flow simulation at $R e_{\tau}=360$ is $12 h^{*} \times$ $6 h^{*} \times 2 h^{*}$, which is approximately twice (in $L_{x}$ and $L_{y}$ ) what was used in Moser et al. (1999, referred to as MKM hereafter) for $R e_{\tau}=395$. In order to use periodic boundary conditions, the computational domain must be large enough to include the largest turbulent structures. This can be checked after the simulation by examination of the two-point correlations of velocity, $R\left(u_{i}^{\prime}\right)$, as shown in figure 2. The two-point correlations fall to zero at maximum separation, demonstrating that the present computational domain is adequate. The total number of grid points used is about 10 million $(256 \times 256 \times 161$ in $x, y, z)$, with spacing of 16.88 and 8.44 wall units in 

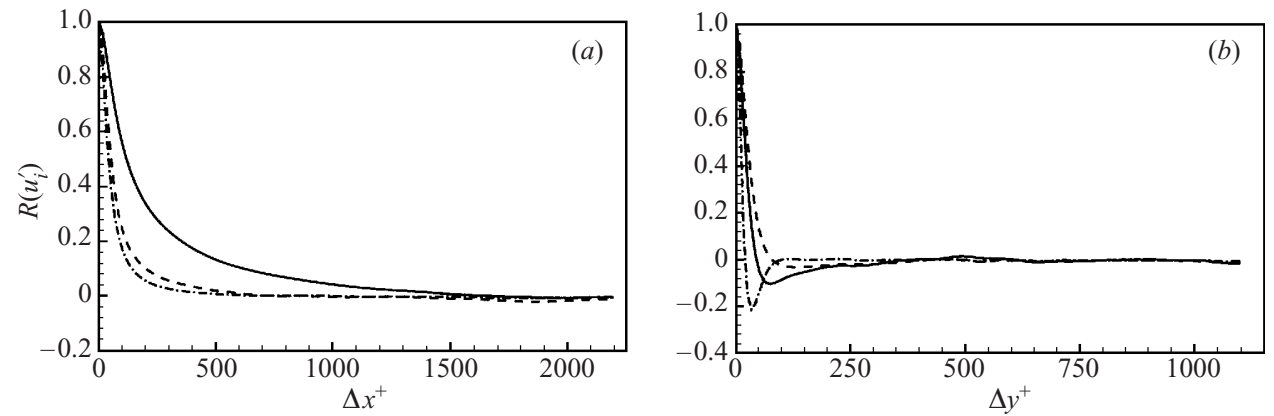

FIgURE 2. Two-point velocity correlations $R\left(u^{\prime}\right)$ (solid line), $R\left(v^{\prime}\right)$ (dashed line) and $R\left(w^{\prime}\right)$ (dash-dotted line) at $z^{+}=17.6$ in Poiseuille flow. $R e_{\tau}=360$. (a) Streamwise, (b) spanwise.

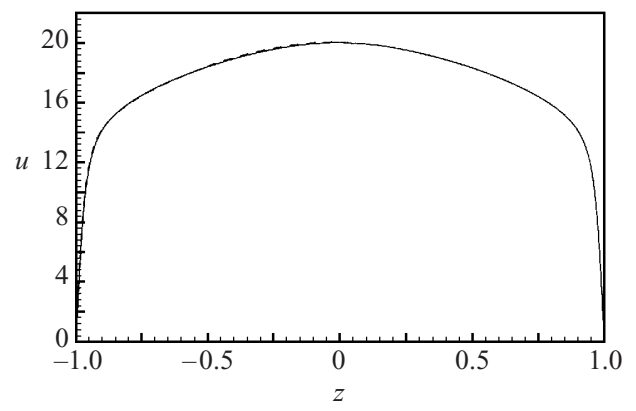

FIGURE 3. Mean velocity of Poiseuille flow (solid line) compared with MKM (dashed line, overlapped by the solid line).

the streamwise and spanwise directions respectively. The computation was carried out with 128 processing elements (PE) on a Cray T3E and required about $12000 \mathrm{PE}$ hours.

The mean velocity and turbulence intensities shown in figures 3 and 4 are in good agreement with MKM. Symmetry of the results about the centreline indicates well-converged statistics. The mean velocity profile collapses to the law of the wall, $u^{+}=(1 / \kappa) \ln y^{+}+B$, with $\kappa=0.41$ and $B=5.0$.

The transport equations of the Reynolds stresses, $R_{i j}=\overline{u_{i}^{\prime} u_{j}^{\prime}}$, can be derived from the continuity and momentum equations as

$$
\frac{\partial R_{i j}}{\partial t}+\bar{u}_{k} \frac{\partial R_{i j}}{\partial x_{k}}=P_{i j}+T_{i j}-D_{i j}-\frac{\partial}{\partial x_{k}}\left(J_{i j k}^{u}+J_{i j k}^{p}+J_{i j k}^{v}\right),
$$

where the terms on the right-hand side are given by

$$
\left.\begin{array}{lll}
P_{i j}=-\left(R_{i k} \frac{\partial \bar{u}_{j}}{\partial x_{k}}+R_{j k} \frac{\partial \bar{u}_{i}}{\partial x_{k}}\right), & T_{i j}=\overline{p^{\prime}\left(\frac{\partial u_{i}^{\prime}}{\partial x_{j}}+\frac{\partial u_{j}^{\prime}}{\partial x_{i}}\right)}, & D_{i j}=\frac{2}{\operatorname{Re} \frac{\partial u_{i}^{\prime}}{\partial x_{k}} \frac{\partial u_{j}^{\prime}}{\partial x_{k}}}, \\
J_{i j k}^{u}=\overline{u_{i}^{\prime} u_{j}^{\prime} u_{k}^{\prime}}, & J_{i j k}^{p}=\overline{p^{\prime} u_{j}^{\prime}} \delta_{i k}+\overline{p^{\prime} u_{i}^{\prime}} \delta_{j k}, & J_{i j k}^{v}=-\frac{1}{\operatorname{Re}} \frac{\partial R_{i j}}{\partial x_{k}} .
\end{array}\right\}
$$

In the above equations, $P_{i j}$ is the production due to mean velocity gradients, $T_{i j}$ is the pressure-strain term, $D_{i j}$ is the 'dissipation' (it is different from the scalar dissipation function $\Phi$, which represents the transfer rate of energy from turbulence to heat) and $J_{i j k}$ is the turbulence flux term, with contribution $J_{i j k}^{u}$ from the turbulence transport 


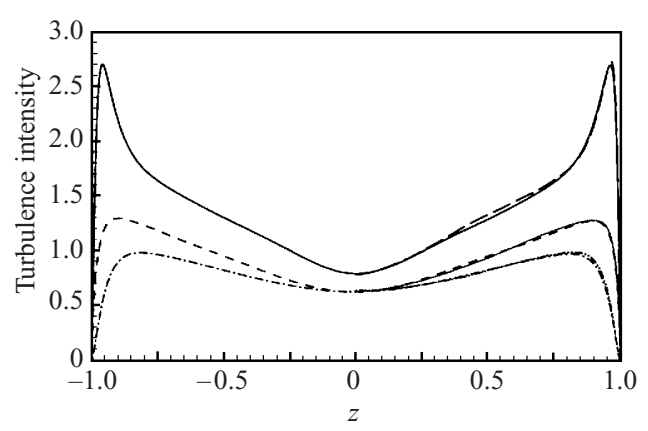

FIGURE 4. Turbulence intensities of Poiseuille flow (solid line: $u_{\mathrm{rms}} / u_{\tau}$, dashed line: $v_{\mathrm{rms}} / u_{\tau}$, dash-dotted line: $w_{\mathrm{rms}} / u_{\tau}$ ), compared on the right with MKM (long dashed line: $u_{\mathrm{rms}} / u_{\tau}$, dash-double-dotted line: $v_{\text {rms }} / u_{\tau}$, dotted line: $w_{\text {rms }} / u_{\tau}$ ).
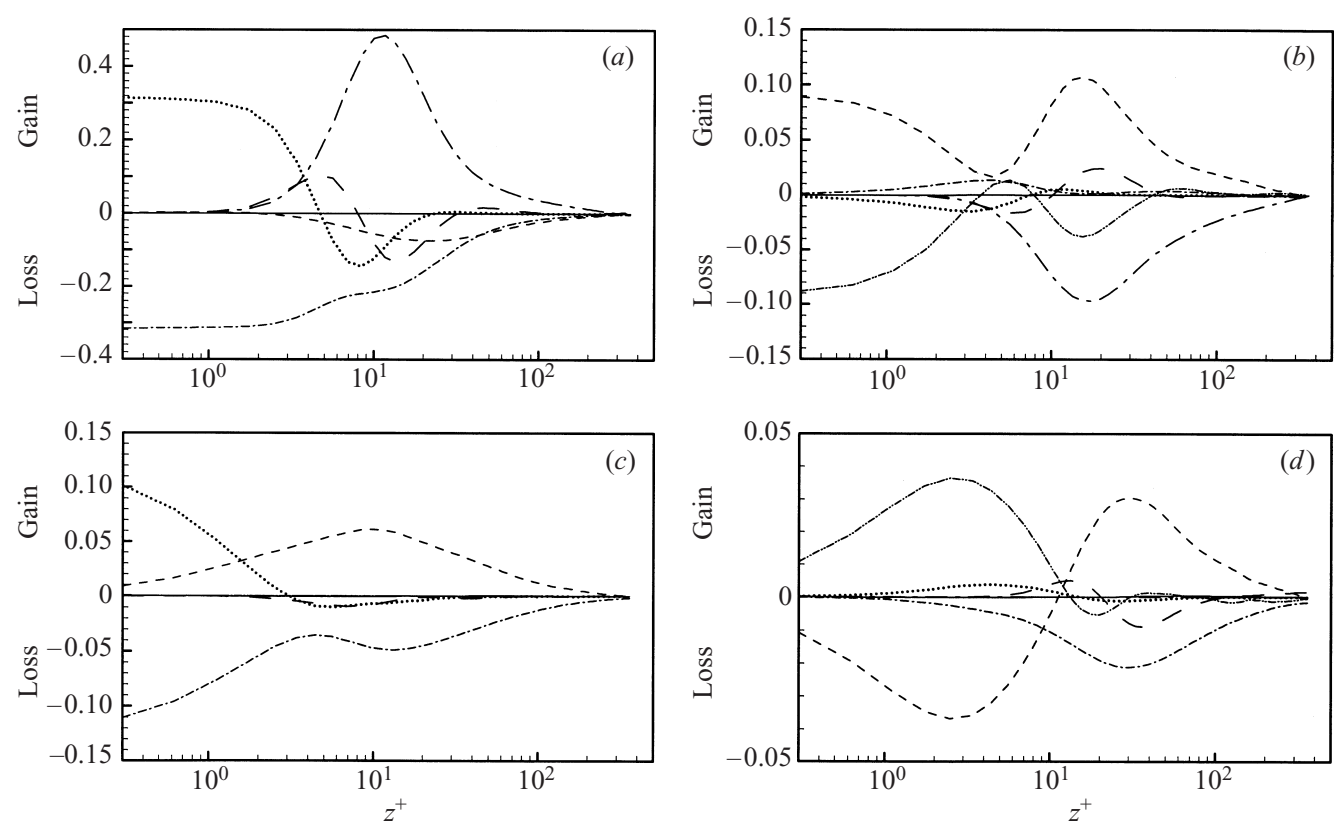

FIGURE 5. Reynolds stress budgets of Poiseuille flow Reynolds stresses. Solid line: imbalance; long dash-dotted line: $P_{i j}$; dashed line: $T_{i j}$; dash-dotted line: $D_{i j}$; long dashed line: $\partial J_{i j k}^{u} / \partial x_{k}$; dash-double-dotted line: $\partial J_{i j k}^{p} / \partial x_{k}$; dotted line: $\partial J_{i j k}^{v} / \partial x_{k}$. (a) $\overline{u^{\prime} u^{\prime}}$, (b) $\overline{u^{\prime} w^{\prime}},(c) \overline{v^{\prime} v^{\prime}}$, (d) $\overline{w^{\prime} w^{\prime}}$.

term, $J_{i j k}^{p}$ from the pressure transport term, and $J_{i j k}^{v}$ from the viscous transport term. After the flow has become statistically stable, the terms on the right-hand side should sum to zero.

Plane Poiseuille and Couette flow are homogeneous in the streamwise and the spanwise directions, with zero Reynolds stress components $R_{12}$ and $R_{23}$. Budgets of the four remaining turbulent stresses have been calculated as a check on our simulation results, as shown in figure 5. The budget balances (sum of all terms on the right-hand side) are of the order of $10^{-4}$. All quantities in figure 5 are normalized using $U_{\text {ref }}^{*}=u_{\tau}^{*}, L_{\text {ref }}^{*}=v^{*} / u_{\tau}^{*}$ (wall scaling). 

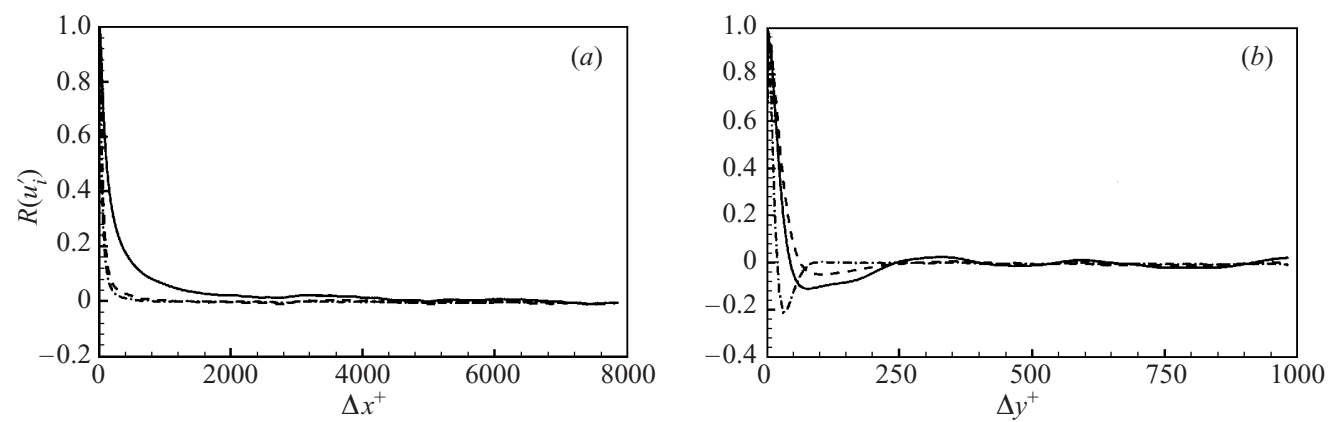

FIgURE 6. Two-point velocity correlations $R\left(u^{\prime}\right)$ (solid line), $R\left(v^{\prime}\right)$ (dashed line) and $R\left(w^{\prime}\right)$ (dash-dotted line) at $z^{+}=15.7$ in Couette flow. $R e_{w}=1300$. (a) Streamwise, $(b)$ spanwise.

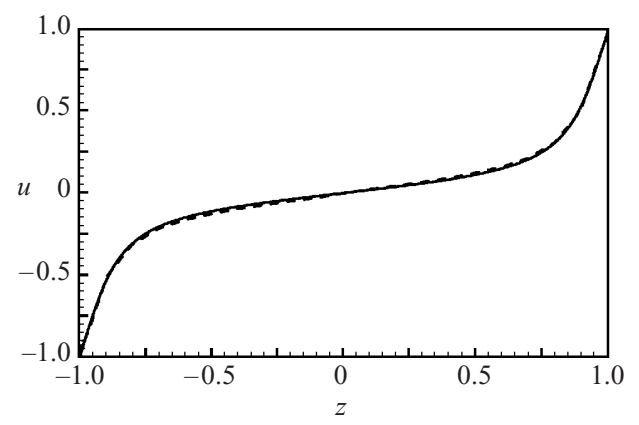

FIGURE 7. Mean velocity of Couette flow (solid line) compared with KBA (dashed line).

\subsection{Plane Couette flow}

A simulation of plane Couette flow at Reynolds number $R e_{w}=1300$ was carried out with a computational domain of $192 h^{*} \times 24 h^{*} \times 2 h^{*}$ and about 42.5 million grid points $(1024 \times 512 \times 81$ in $x, y, z)$. The simulation was run with 128 PEs on a Cray T3E, requiring about 25000 PE hours. The grid spacing was 15.38 and 7.69 wall units in the streamwise and spanwise directions respectively. The computational box is large enough to allow periodic boundary conditions to be applied in both streamwise and spanwise directions, as demonstrated by the streamwise and spanwise two-point correlations shown in figure 6 for a wall-normal position close to the kinetic energy maximum.

DNS of Couette flow is more difficult than Poiseuille flow, since very long structures exist in the central core of the channel (Komminaho et al. 1996). Hu \& Sandham (2001) found that in the spanwise direction the large structure in Couette flow scales on channel width, and in the streamwise direction it scales on wall units. The streamwise length scale of large structures in Couette flow is much larger than that in Poiseuille flow.

Figure 7 shows the mean velocity profile found for plane Couette flow; it is in good agreement with the $R e_{w}=1300$ simulation of Kristoffersen et al. (1993, referred to as KBA hereafter), and has a typical S-shaped mean velocity profile, leading to a nonzero mean velocity gradient at the centreline. The non-dimensional centreline velocity gradient is 0.1924 for $R e_{w}=1300$; another simulation at $R e_{w}=3400$ gives 0.1980 . The DNS of Komminaho et al. (1996) for Reynolds number $R e_{w}=750$ gave a value of 0.18 . All these gradients are well within the range 0.15 to 0.3 given by Tillmark 


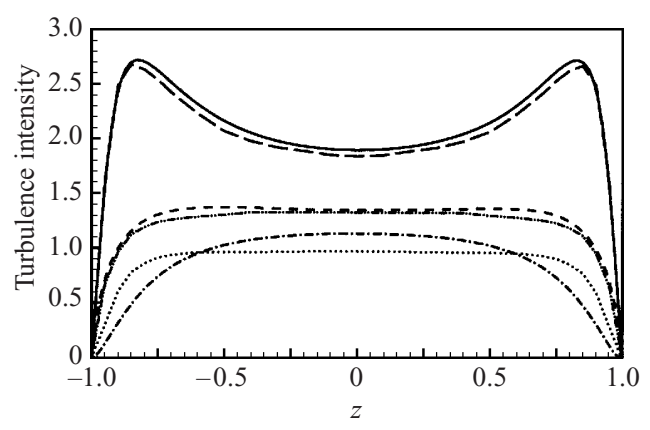

FIGURE 8. Turbulence intensities $u_{\text {rms }} / u_{\tau}, v_{\text {rms }} / u_{\tau}, w_{\text {rms }} / u_{\tau}$, for Couette flow, compared with KBA. See figure 4 for legend.
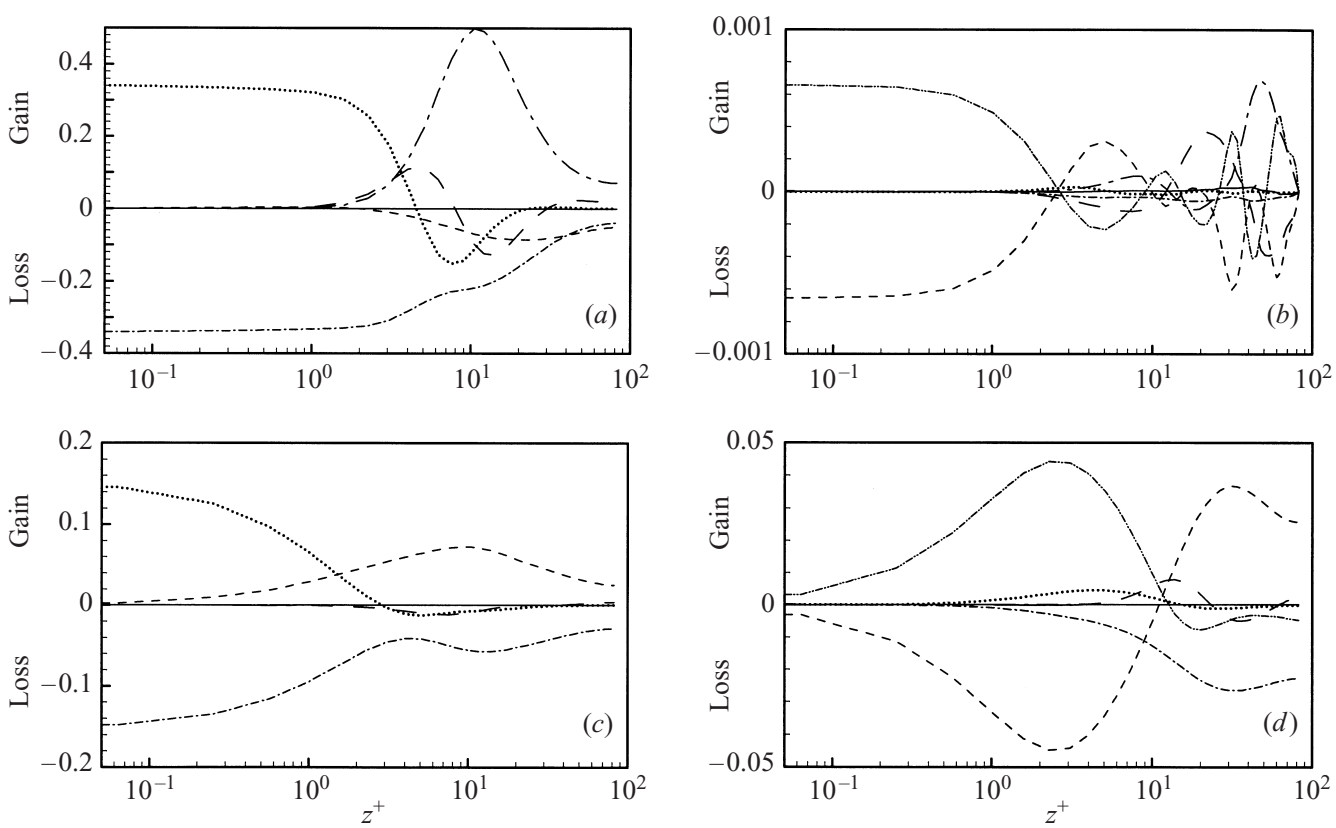

FIGURE 9. Reynolds stress budgets of Couette flow Reynolds stresses. (a) $\overline{u^{\prime} u^{\prime}}$, (b) $\overline{u^{\prime} w^{\prime}}$, (c) $\overline{v^{\prime} v^{\prime}},(d) \overline{w^{\prime} w^{\prime}}$. Plots terminate at the centreline. See figure 5 for legend.

\& Alfredsson (1993) from experimental data. The finite mean velocity gradient gives Couette flow a finite shear stress at the centreline, which is necessary for non-zero Reynolds stress production (as shown in figure $9 a$ ).

Turbulence intensities for plane Couette flow are given in figure 8, with all quantities normalized by $u_{\tau}^{*}$. Results of KBA are also plotted with thin lines for comparison. The streamwise and spanwise turbulence intensities of KBA are close to the present results in the near-wall region, but smaller elsewhere; the differences are almost certainly caused by the coarser resolution and smaller box used in KBA, as explained in $\mathrm{Hu}$, Morfey \& Sandham (2002).

Budgets of Couette flow Reynolds stresses are shown in figure 9. All quantities are scaled using wall variables. Very good balances are achieved, demonstrating high accuracy in the simulations. The maximum imbalance is less than $2 \times 10^{-4}$. 


\section{The acoustic sources of channel flow}

\subsection{Contributions to the source wavenumber-frequency spectrum}

The source wavenumber-frequency spectrum, $S_{\Gamma}(\boldsymbol{k}, f)$, defined in (42) and (43) can be expressed as

$$
S_{\Gamma}(\boldsymbol{k}, f)=\lambda^{2} M^{4} S_{m}+M^{2} e_{\alpha} e_{\beta} S_{\alpha \beta}+M^{4} e_{\alpha} e_{\beta} e_{\gamma} e_{\delta} S_{\alpha \beta \gamma \delta}+C \quad(\alpha, \beta=1,2),
$$

where $S_{m}, S_{\alpha \beta}$ and $S_{\alpha \beta \gamma \delta}$ are order-1 wavenumber-frequency spectra associated respectively with the monopole, dipole and quadrupole terms in (36), and $C$ represents cross-terms (e.g. dipole-monopole, dipole-quadrupole). Some but not all of the crossterms vanish at zero wavenumber, as a consequence of symmetry (Morfey 1999). Attention is focused here on the first three terms, since if one of these dominates the other two, the cross-terms will make only a minor contribution.

Expressions for the $S_{m}, S_{\alpha \beta}$ and $S_{\alpha \beta \gamma \delta}$ spectra follow from substituting equations (28)-(30) into (36):

$$
\left.\begin{array}{l}
S_{m}(\boldsymbol{k}, f)=(2 \pi f)^{2} S_{\Psi}(\boldsymbol{k}, f), \\
S_{\alpha \beta}(\boldsymbol{k}, f)=(2 \pi f)^{2} S_{f_{f} f_{\beta}}(\boldsymbol{k}, f), \\
S_{\alpha \beta \gamma \gamma}(\boldsymbol{k}, f)=(2 \pi f)^{4} S_{t_{\alpha \beta} t_{\gamma \delta}}(\boldsymbol{k}, f) ;
\end{array}\right\}
$$

here $\Psi$, defined in (30), is the dissipation averaged over the channel width, and $f_{\alpha}$, $t_{\alpha \beta}$ are defined in (29) and (28). The notation $S_{\Psi}$ refers to the power spectrum of $\Psi$, and $S_{p q}$ refers to the cross-power spectrum of $p$ and $q$.

Some simplification results from the fact that at zero wavenumber, certain of the $S_{\alpha \beta}$ and $S_{\alpha \beta \gamma \delta}$ components vanish, by symmetry, for plane channel flow: specifically, those with odd numbers of indices 1 and 2 (Morfey 1999). Examples are

$$
S_{12}(\mathbf{0}, f)=0, \quad S_{1112}(\mathbf{0}, f)=0 .
$$

The surviving contributions to $S_{\Gamma}(\mathbf{0}, f)$ are given below (with arguments suppressed):

$$
\left.\begin{array}{ll}
\text { monopole: } & S_{\Gamma, m}=(2 \pi f)^{2} \lambda^{2} M^{4} S_{\Psi}, \\
\text { dipole: } & S_{\Gamma, d}=(2 \pi f)^{2} M^{2}\left\{e_{1}^{2} S_{f_{1} f_{1}}+e_{2}^{2} S_{f_{2} f_{2}}\right\}, \\
\text { quadrupole: } & S_{\Gamma, q}=(2 \pi f)^{4} M^{4}\left\{e_{1}^{4} S_{t_{11} t_{11}}+2 e_{1}^{2} e_{2}^{2} S_{t_{12} t_{12}}+e_{2}^{4} S_{t_{22} t_{22}}\right\} .
\end{array}\right\}
$$

When these terms are substituted in (43), it is clear that the wall-stress dipole radiation dominates in the low-Mach-number limit, with

$$
S_{p, d}(\boldsymbol{x}, f) \approx \frac{\pi^{2} f M}{|\boldsymbol{x}|}\left\{e_{1}^{2} S_{f_{1} f_{1}}(\mathbf{0}, f)+e_{2}^{2} S_{f_{2} f_{2}}(\mathbf{0}, f)\right\},
$$

provided the zero-wavenumber spectral densities $S_{f_{1} f_{1}}(\mathbf{0}, f)$ and $S_{f_{2} f_{2}}(\mathbf{0}, f)$ are not both zero. This last issue will be addressed in $\$ \S 6.3$ and 6.5 below, for Poiseuille and Couette flow respectively.

\subsection{Compactness of the equivalent dipole and quadrupole sources}

Before proceeding further, it is necessary to check that two-point correlations of the equivalent source distribution $\Gamma(\boldsymbol{x}, t)$ decay rapidly enough, as a function of separation, that they fall effectively to zero within the box $L_{1} \times L_{2}$. Here $\Gamma(\boldsymbol{x}, t)$ is the Fourier transform of $\Gamma(\boldsymbol{x}, f)$ defined in (36):

$$
\Gamma(\boldsymbol{x}, t)=M^{2} e_{\alpha} e_{\beta} \ddot{t}_{\alpha \beta}+M e_{\alpha} \dot{f}_{\alpha}+\dot{q},
$$



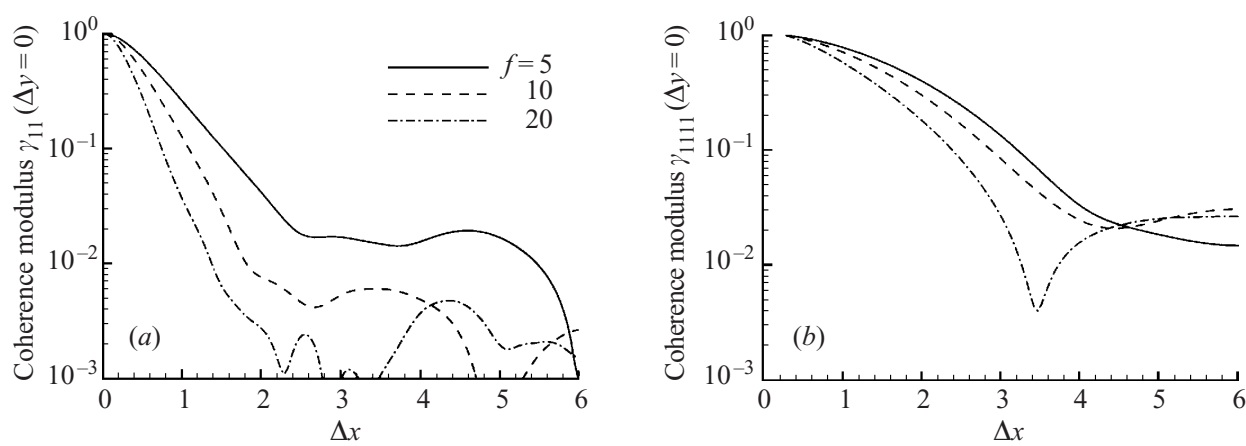

FIGURE 10. Decay of coherence with streamwise separation, for the streamwise component of the wall-shear dipole $(a)$ and the turbulent Reynolds stress averaged across the channel ( $x x$-quadrupole) (b) in Poiseuille flow $\left(R e_{\tau}=360\right)$. Results are shown for three non-dimensional frequencies $(f=5,10,20)$.
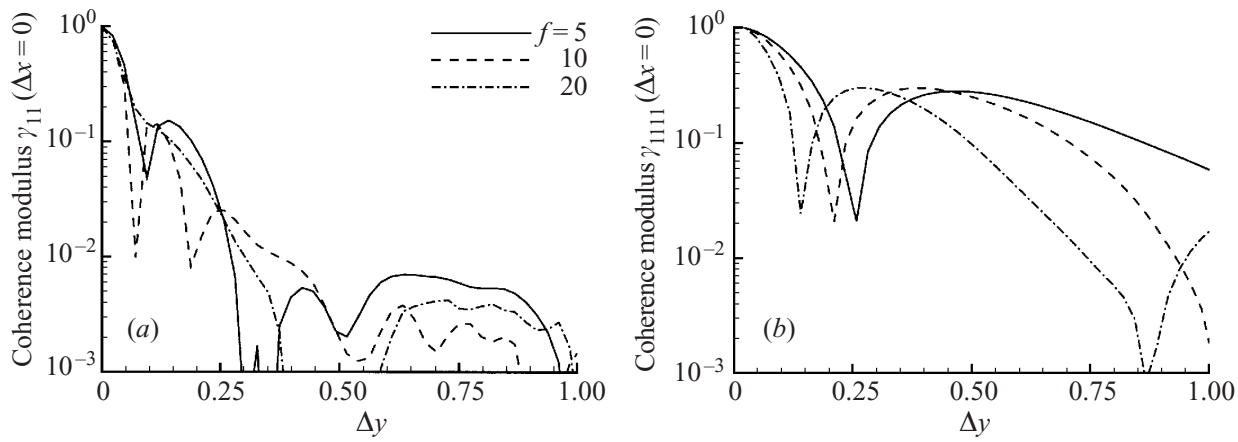

FIGURE 11. As figure 10, but for spanwise separation.

with dots denoting time derivatives. In this section we explore the two-point correlation properties of the streamwise dipole term $\dot{f}_{1}$ and the streamwise quadrupole term $\ddot{t}_{11}$.

Wavenumber-frequency spectra for these quantities, denoted in (60) above by $S_{11}(\boldsymbol{k}, f)$ and $S_{1111}(\boldsymbol{k}, f)$, are first formed from the DNS output as described in $\S 3.5$. Two-point cross-spectra follow via two-dimensional spatial Fourier transformation. If $S_{11}(\Delta x, \Delta y, f)$ denotes the cross-spectrum of $\dot{f}_{1}$ evaluated at points with separation $(\Delta x, \Delta y)$, then

$$
g_{11}(\Delta x, \Delta y, f)=S_{11}(\Delta x, \Delta y, f) / S_{11}(0,0, f) \quad(\text { streamwise dipole })
$$

is the complex frequency-domain coherence function for $\dot{f}_{1}$, with magnitude 1 at zero separation. The corresponding coherence function for $\ddot{t}_{11}$ is defined similarly. Figure 10 shows how the coherence magnitudes $\gamma=|g|$ decay with streamwise separation $\Delta x$ (at $\Delta y=0$ ), for Poiseuille flow at $R e_{\tau}=360$ and three different frequencies. Figure 11 presents the corresponding plots for spanwise separation $\Delta y$ (at $\Delta x=0$ ).

It is clear from these comparisons that the wall-dipole coherence falls off faster than the Reynolds-stress quadrupole coherence, in both the streamwise and spanwise directions. The streamwise decay in the dipole case is approximately exponential. Note that the $\Delta x$ scale extends to half the box length $(\Delta x=6)$; the $\Delta y$ scale is truncated at one-sixth of the box length $(\Delta y=1)$, in order to show the rapid spanwise decay in more detail. In all cases, the coherence magnitude decays to near zero (less than $3 \times 10^{-2}$ ) within half the box dimensions. 

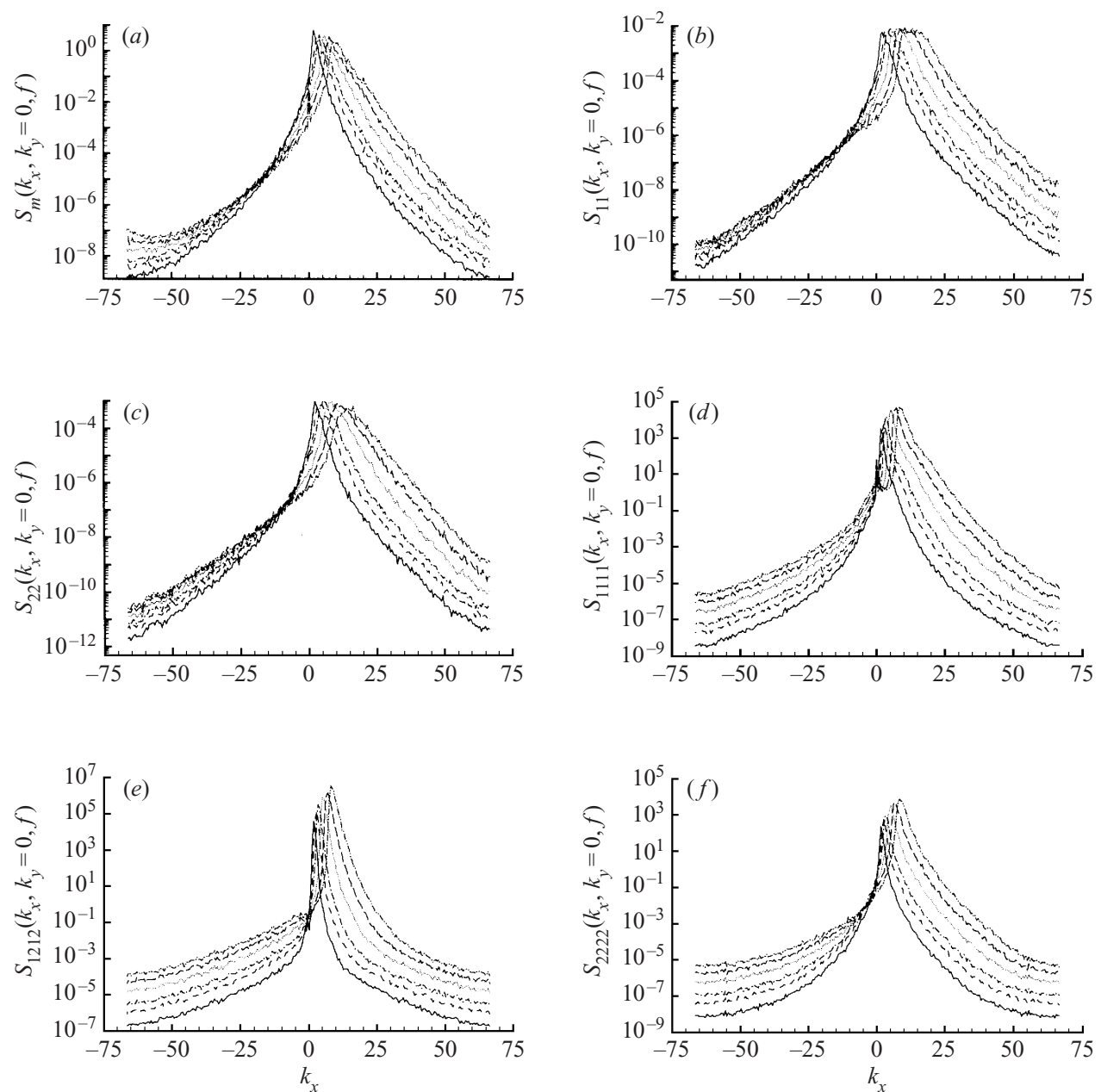

FIGURE 12. Wavenumber-frequency spectral densities of Poiseuille flow sound radiation sources in equation (60), plotted against streamwise wavenumber $k_{x}$ with $k_{y}=0$. Solid line: $f=5$; dashed line: $f=7.5$; dash-dotted line: $f=10$; dotted line: $f=15$; long dashed line: $f=20$; dash-double-dotted line: $f=25$. $R e_{\tau}=360$. ( $a$ ) Monopole, $(b)$ streamwise dipole, $(c)$ spanwise dipole, $(d) x x$-quadrupole, (e) $x y$-quadrupole, $(f)$ yy-quadrupole.

The faster coherence decay in both directions for the fluctuating wall shear stress, compared with the Reynolds stress averaged across the channel, makes it possible to estimate the shear-stress correlation area from the DNS results with some confidence at each frequency. In other words, the zero-wavenumber information on $S_{\alpha \beta}(\boldsymbol{k}, f)$ required by (63) is accessible with the present box size. Further evidence to support this statement is presented in $\S 6.7$.

\subsection{Source spectra for plane Poiseuille flow}

Figure 12 presents numerical results for Poiseuille flow at $R e_{\tau}=360$, equivalent to $R e=14500$ based on centreline velocity and channel width. The spectral factors $S_{m}, S_{\alpha \beta}$ and $S_{\alpha \beta \gamma \delta}$ defined in (59) and (60) are plotted as functions of the streamwise wavenumber, with the spanwise wavenumber set equal to zero. Throughout the rest of $\S 6$ we use $x$ and $y$ to label streamwise and spanwise directions, but retain Cartesian subscripts $(1,2)$ for source terms; thus the $x y$-quadrupole spectra in figure $12(e)$ refer to the $S_{1212}(\boldsymbol{k}, f)$ term in $(60)$. 

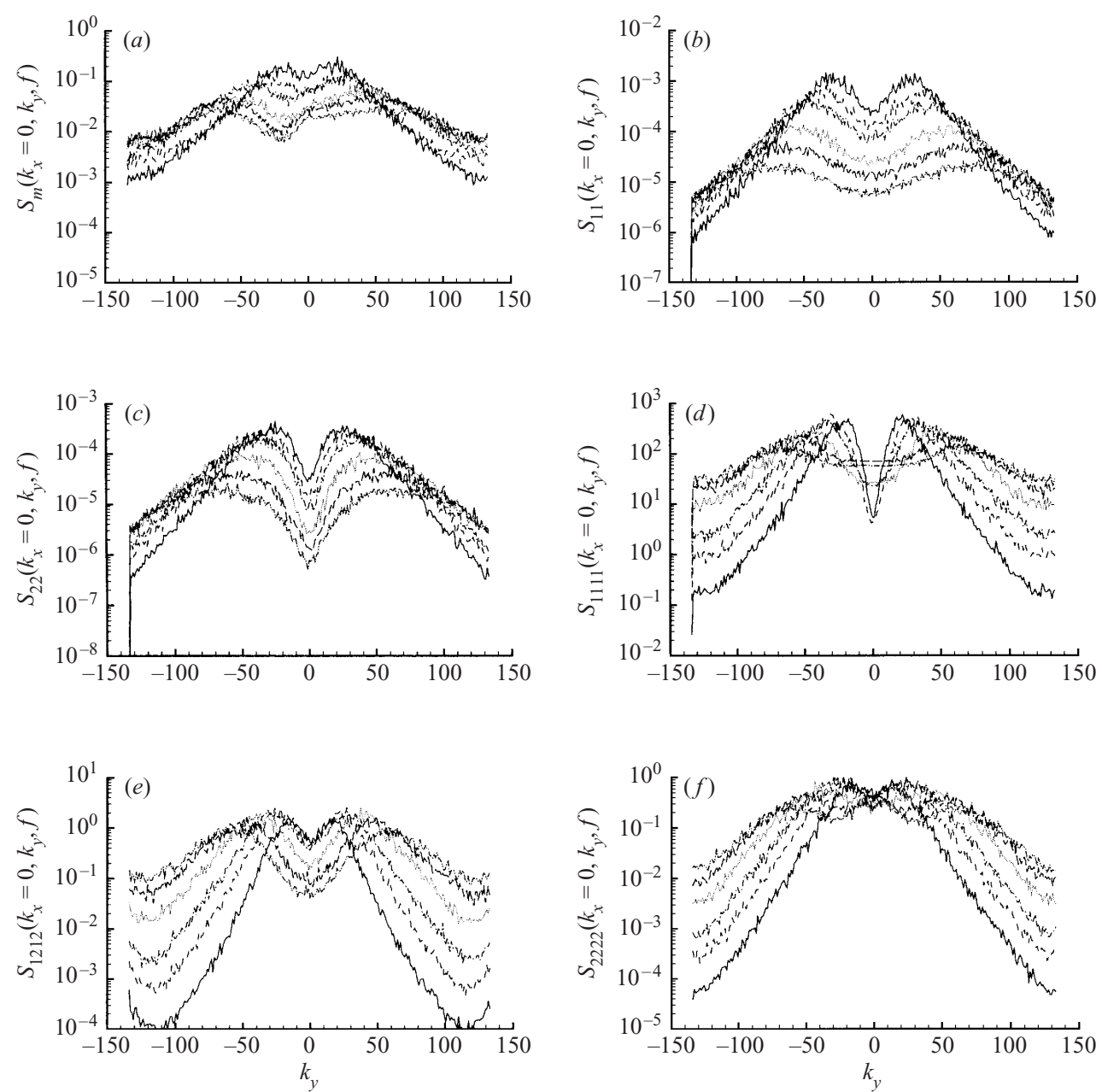

FIGURE 13. As figure 12 but for spanwise wavenumber $k_{y}$ with $k_{x}=0$.

Shown on each plot are six different scaled frequencies $f$ (scaled on the friction velocity $u_{\tau}^{*}=\left(\tau_{w}^{*} / \rho^{*}\right)^{1 / 2}$ and channel half-width $\left.h^{*}\right)$. Convective peaks in the $k_{x}$ spectra are clearly visible, as discussed in $\mathrm{Hu}$ et al. (2002); the peak wavenumber is positive, and varies linearly with frequency, defining a convection velocity $u_{c}$. However, the most important observation for the present study is that in the sub-convective region (i.e. $\left.\left|k_{x}\right|<2 \pi f / u_{c}\right)$, the wavenumber spectra remain finite as the streamwise wavenumber approaches zero. This means that the incompressible simulation can provide estimates of the sound radiation from each type of source, in the limit $M \ll 1$.

The small increase at large negative $k_{x}$ in the monopole spectral density for high frequencies is an effect of numerical resolution. Numerical experiments have shown that higher resolution will give monotonically decreasing spectra. The peaks observed at zero wavenumber for $f=20$ and 25 (monopole, $x x$-quadrupole) will be discussed in $\S 6.4$, in the context of numerical noise.

Figure 13 shows the same spectral factors plotted against spanwise wavenumber $k_{y}$, with $k_{x}$ now set equal to zero. The $y$-dipole and the $x x$-quadrupole both show distinct minima in their $k_{y}$ power spectra at $k_{y}=0$, but their zero-wavenumber values are well above the numerical noise floor in most cases (except for the $x x$-quadrupole at 

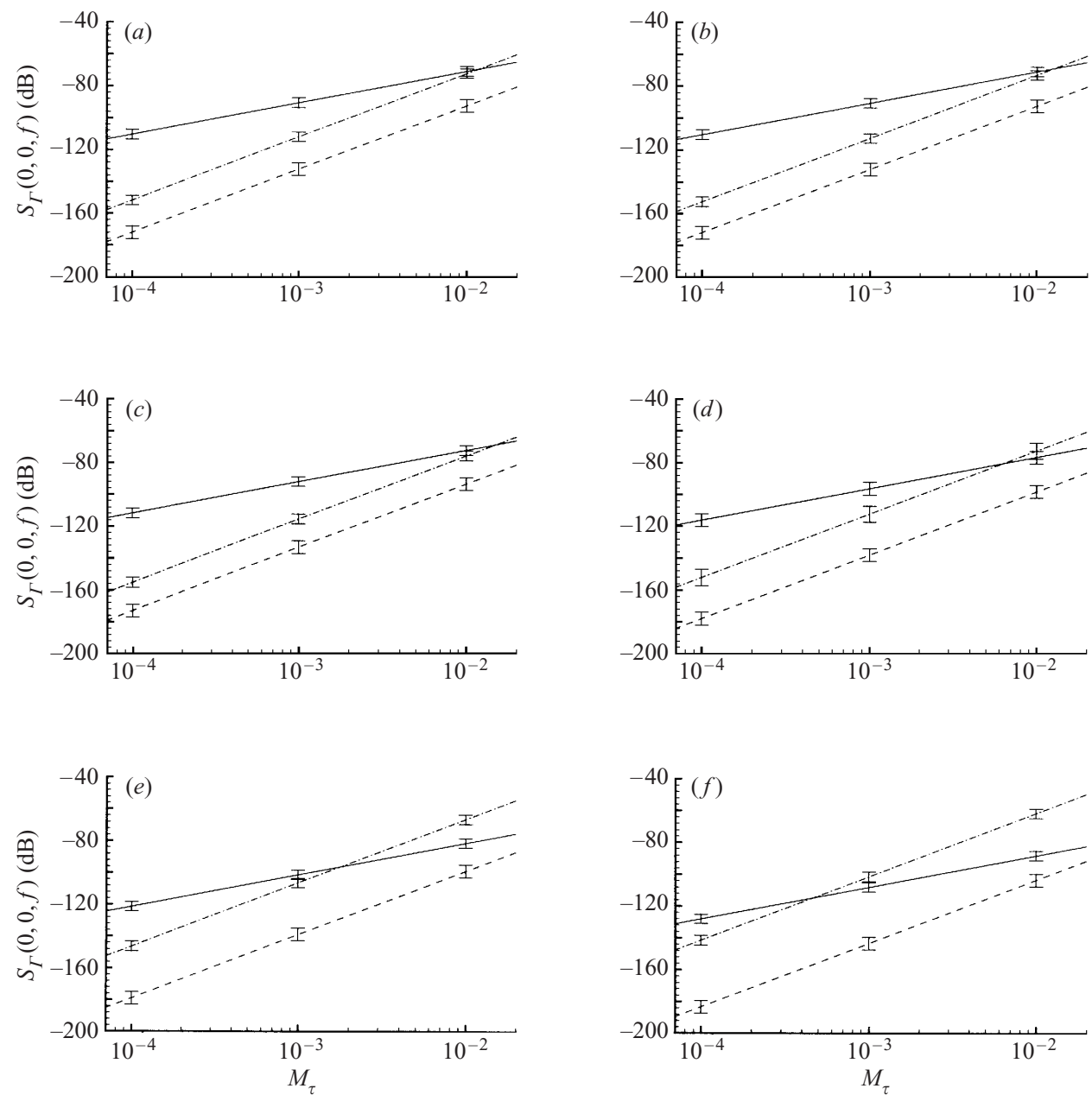

FiguRE 14. Contribution of different source terms to far-field sound radiation intensity of plane Poiseuille flow in the streamwise direction. Error bars are estimated from the uncertainty of spectra near $(0,0)$ wavenumbers. Dashed line: monopole; solid line: $x$ dipole; dash-dotted line: $x x$ quadrupole. $R e_{\tau}=360$. (a) $f=0.63,(b) f=1.25,(c) f=2.5,(d) f=5,(e) f=10,(f) f=20$.

$f=20$ and 25 , noted above). Evidence for the robustness of these low-wavenumber estimates will be presented in $\S 6.7$.

For radiation in the streamwise direction with air as the fluid $(\lambda=0.4)$, the relative contributions of the $\lambda^{2} S_{m}$ monopole term, the $S_{1111}$ streamwise quadrupole term, and the $S_{11}$ streamwise dipole term to $S_{\Gamma}(\mathbf{0}, f)$ are plotted in figure 14 as a function of the Mach number $M_{\tau}$ (based on friction velocity). Note that the monopole and quadrupole contributions both vary as $M^{4}$, in contrast to the $M^{2}$ dependence of the dipole radiation; see equation (59) and the comments that follow.

Figure 14 shows that at sufficiently low Mach numbers, the dipole radiation is dominant over the whole frequency range. For $R e_{\tau}=360$ and frequencies up to $f=20$, this requires $M_{\tau}<4 \times 10^{-4}$. Since $M_{\max }$ (the centreline Mach number) is approximately $20 M_{\tau}$ in this case, it is clear that quadrupole radiation is insignificant only for flows of very low Mach number, of order $M_{\max }=0.01$ or less. However, the monopole radiation due to fluctuating dissipation in air can be neglected entirely in 


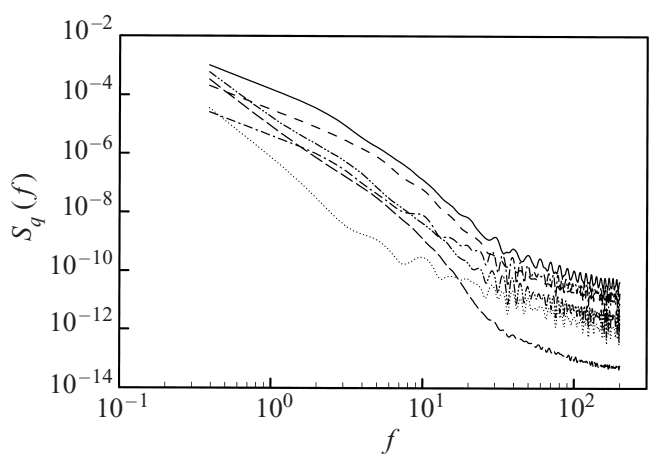

FIGURE 15. Normalized wavenumber-frequency spectra of the source terms $S_{f_{1} f_{1}}$, $S_{\Psi}$, etc. defined in (60), evaluated at zero wavenumber for plane Poiseuille flow at $R e_{\tau}=360$. Solid line: $x$-dipole; dashed line: $y$-dipole; dash-dotted line: monopole; dotted line: $x x$-quadrupole; long-dash line: $x y$-quadrupole; dash-double-dotted line: $y y$-quadrupole. For definition of $\hat{S}_{q}(f)$, see $(66)$.

the streamwise direction at this Reynolds number; it follows the same $M$ dependence as the quadrupole radiation, but lies $20 \mathrm{~dB}$ below it at low frequencies $(f<2.5)$, increasing to $40 \mathrm{~dB}$ below it at $f=20$. Compare figure 16 below for spanwise radiation, where the gap is much less.

\subsection{Relative contributions of the various source terms versus frequency}

It is helpful to examine the various contributions to $S_{\Gamma}$ in the form of frequency spectra, rather than at selected frequencies as in figure 14. However, this raises the issue of numerical noise. It is clear from (62) that the terms in $S_{\Gamma}$ are related to more fundamental quantities like $S_{\Psi}$ by weighting factors that depend on Mach number and frequency. Let $\Psi$, or any of the other fluctuating quantities $\left(f_{1}, f_{2}, t_{11}, t_{12}, t_{22}\right)$ whose spectra appear in (62), be denoted by $q(x, y, t)$. Spectral analysis of $q$ in wavenumber and frequency, as required for the acoustic calculation, amounts to partitioning the signal energy into a three-dimensional array of bins, some of which may contain a very small fraction of the total. In order to assess the numerical limitations of the spectral analysis process, we define the normalized spectral quantity

$$
\hat{S}_{q}(f)=\frac{1}{\left\langle q^{2}\right\rangle} S_{q}(0,0, f),
$$

i.e. the zero-wavenumber spectral density divided by the mean-square signal $\dagger$, for each of the $q$ data files generated by the flow simulation. Figure 15 shows the various $\hat{S}_{q}$ plotted versus frequency for Poiseuille flow. Once $\hat{S}_{q}$ falls below $10^{-9}$, there are signs of numerical inaccuracy: there appears to be a numerical noise floor at around $10^{-9}$, possibly set by round-off errors in the threefold discrete Fourier transformation. Reliable spectral information on $S_{q}(0,0, f)$ is generally limited to $f<25$, with a slightly lower cutoff (around $f=10-15$ ) applying to the monopole and $x x$-quadrupole: this explains the spikes at $k_{x}=0$ seen in the wavenumber spectra of figure $12(a)$ and $12(d)$, at $f=20$ and 25 .

Keeping the last observation in mind, we turn to the final three figures of this section on Poiseuille flow. Figure 16 focuses on source terms that contribute to radiation in the spanwise direction: plotted versus frequency are the three relevant

$\dagger$ Note that $\hat{S}_{q}(f)$ is closely related to the correlation area of $q(x, y, t)$, since $(2 \pi)^{2} \int_{-\infty}^{\infty} \hat{S}_{q}(f) \mathrm{d} f=A_{\text {corr }}=\iint_{-\infty}^{\infty} \hat{R}_{q}(\xi, \eta) \mathrm{d} \xi \mathrm{d} \eta$, where $\hat{R}_{q}$ is the normalized cross-correlation function of $q$, evaluated at zero time delay and spatial separation $(\xi, \eta)$. 


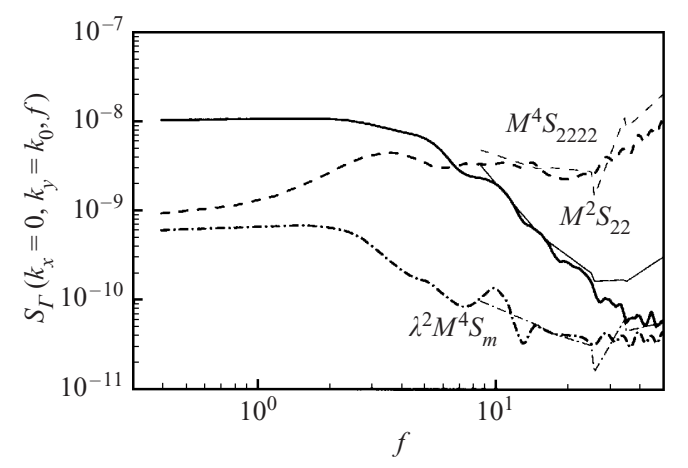

FIGURE 16. Comparison of acoustic source spectra for radiation in the spanwise direction, for plane Poiseuille flow at $R e_{\tau}=360$ (scaled to $M_{\tau}=0.01$ ). Solid line: $y$-dipole; dashed line: $y y$-quadrupole; dash-dotted line: monopole. The main curves (with thick lines) correspond to $k_{0}=0$; in the shorter curves (with thin lines), the wavenumber spectra have been evaluated at a finite wavenumber, corresponding to $k_{0}=0.122 f$.

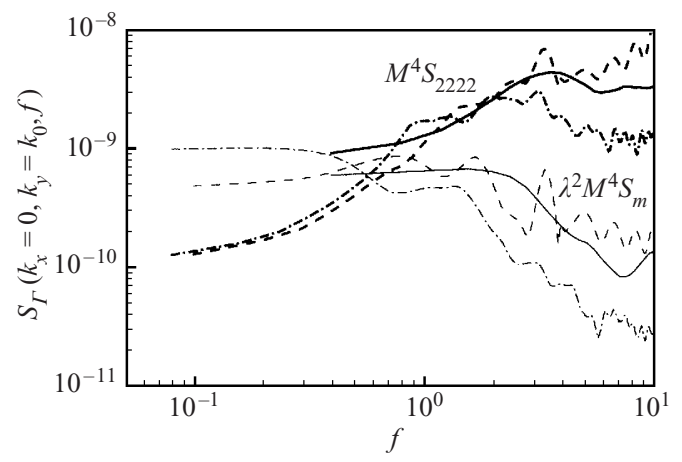

FIGURE 17. Further comparison of acoustic source spectra for radiation in the spanwise direction, at three different Reynolds numbers in plane Poiseuille flow (scaled to $M_{\tau}=0.01$ ): $y y$-quadrupole (thick lines) versus monopole (thin lines). $R e_{\tau}=360$ (solid line), 180 (dashed line), 135 (dash-dotted line). Wavenumber spectra evaluated at zero wavenumber. Compare figure 16.

terms of (59), with the Mach number set equal to $10^{-2}$, and with $\lambda=0.4$. At this low Mach number, the $y$-dipole term is dominant over the whole frequency range up to $f=5$. Each of the other two terms ( $y y$-quadrupole and monopole) has an additional $M^{2}$ weighting; it is interesting to see that the monopole contribution is almost as large as the spanwise quadrupole at low frequencies $(f<1)$, but falls off rapidly at high frequencies. The same monopole-quadrupole comparison is continued in figure 17, for three different Reynolds numbers: progressive reduction of $R e_{\tau}$ below 360 clearly enhances the low-frequency monopole contribution, while leaving the spanwise Reynolds stress contribution largely unchanged.

Lastly figure 18 presents frequency spectra for the zero-wavenumber component of the dipole source term $S_{11}$ that dominates sound radiation in the streamwise direction, again at three different Reynolds numbers. The peakiness apparent beyond $f=4$ at the lower Reynolds numbers, particularly at $R e_{\tau}=180$, is probably a numerical artifact; the same applies to the lower Reynolds numbers in figure 17. Significantly, the flat region of the dipole source spectrum extends to higher scaled frequencies $\left(f=f^{*} h^{*} / u_{\tau}^{*}\right)$ as $R e_{\tau}$ increases; figure 17 shows signs of a similar trend for the monopole spectrum.

A feature of this plot, and also figure 16, is the inclusion of additional curves for 


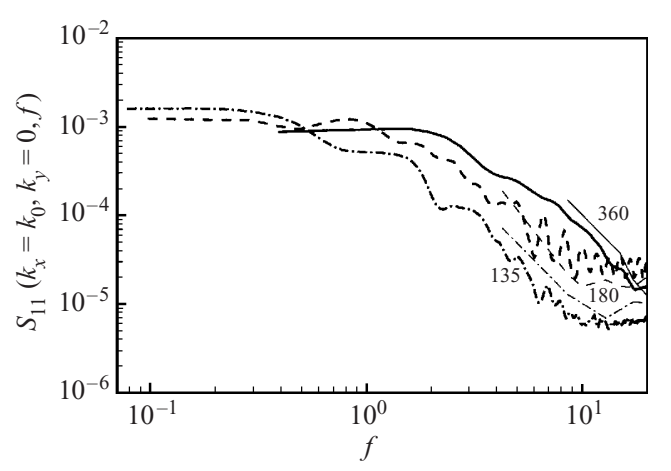

FIGURE 18. Streamwise-radiation frequency spectrum of the streamwise-dipole source term $S_{11}$ in equation (59), plotted at three different Reynolds numbers for plane Poiseuille flow: $R e_{\tau}=360$ (solid line), 180 (dashed line), 135 (dash-dotted line). The main curves (with thick lines) correspond to $k_{0}=0$; in the shorter curves (with thin lines), the wavenumber spectra have been evaluated at a finite wavenumber, corresponding to $k_{0}=0.061 f$.

finite $k_{0}$ : these are 'compressible' predictions, obtained by interpolating the threedimensional $\left(k_{x}, k_{y}, f\right)$ source spectra at $\left(k_{x}=k_{0}=2 \pi f M_{\tau}, k_{y}=0\right)$ for streamwise radiation, and at $\left(k_{x}=0, k_{y}=k_{0}\right)$ for spanwise radiation. The quoted $k_{0}$ values correspond to a bulk mean Mach number of 0.34 in figure 16 and 0.17 in figure 18; very little difference appears in the source spectra, as compared with the zero Mach number limit.

\subsection{Source spectra for plane Couette flow}

Figures 19 and 20, calculated for Couette flow at $R e_{w}=1300$ based on wall velocity, correspond to figures 12 and 13 for Poiseuille flow. The single convective peak seen in the $k_{x}$ spectra of figure 12 is replaced by a double hump, consistent with the motion of the two walls in opposite directions (Hu et al. 2002). Note that for Couette flow, all frequencies are scaled on the wall velocity, rather than the friction velocity.

With respect to the zero-wavenumber limit, the same conclusion follows as before: all the source spectra remain finite through $k_{x}=0, k_{y}=0$. At high frequencies $(f>0.25)$, the monopole and $x x$-quadrupole $k_{x}$ spectra show a pronounced peak at zero wavenumber (also visible at $f=20$ and 25 for Poiseuille flow). The corresponding $k_{y}$ spectra (spanwise) are noticeably lacking in symmetry, which suggests either insufficient run time or a residual influence of finite spanwise box size. A similar phenomenon can be seen in figure 13 for Poiseuille flow.

For radiation in the streamwise direction in air $(\lambda=0.4)$, figure 21 shows how the relative contributions to $S_{\Gamma}(\mathbf{0}, f)$ from the monopole spectrum $S_{m}$, the $x x$-quadrupole spectrum $S_{1111}$, and the $x$-dipole spectrum $S_{11}$ vary with Mach number. Note that the horizontal axis is the wall Mach number $M_{w}$, rather than $M_{\tau}$. Over the frequency range for which reliable data are available (up to $f=0.4$ ), the wall-shear dipole radiation is dominant for Mach numbers less than $10^{-2}$ over the entire frequency range, at the Reynolds number of the present simulation $\left(R e_{w}=1300\right)$. The dipoledominated range extends to higher Mach numbers as the frequency is reduced; for example it reaches up to $M_{w}=0.1$ at $f=0.1\left(f^{*}=0.1 u_{w}^{*} / h^{*}\right)$.

Finally, figure 22 for Couette flow may be compared with figure 18 for Poiseuille flow. It shows frequency spectra for radiation in the streamwise direction from the wall-shear dipoles ( $S_{11}$ source term), at two values of $R e_{w}$. As with the corresponding Poiseuille flow $S_{11}$ spectrum, the flat region extends to higher scaled frequencies 

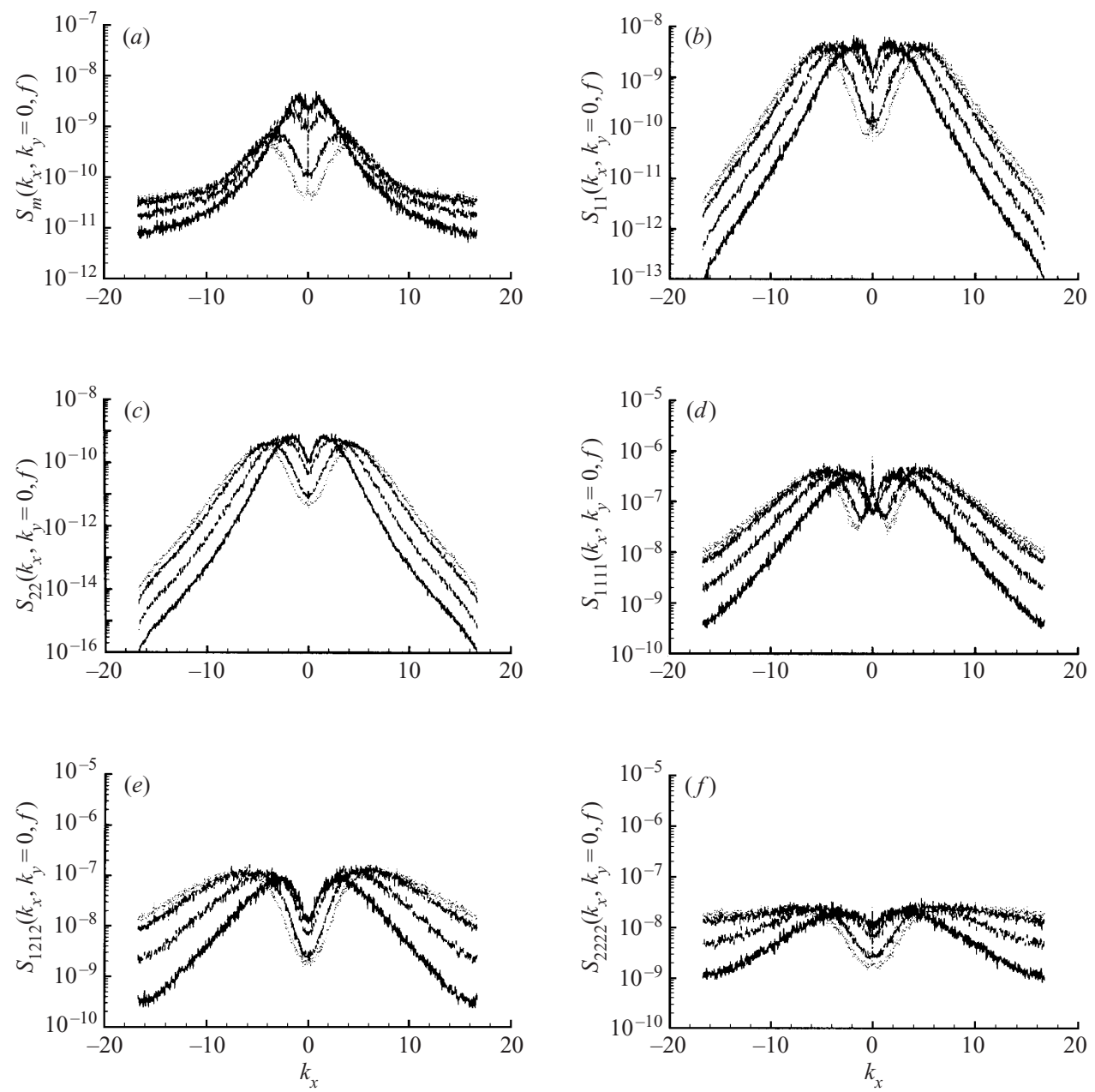

FigURE 19. Wavenumber-frequency spectral densities of Couette flow sound radiation sources, plotted against streamwise wavenumber $k_{x}$ with $k_{y}=0$. Solid line: $f=0.1$; dashed line: $f=0.25$ dash-dotted line: $f=0.4$; dotted line: $f=0.5$. $R e_{w}=1300$. $(a)$ Monopole, $(b)$ streamwise dipole, $(c)$ spanwise dipole, $(d) x x$-quadrupole, $(e) x y$-quadrupole, $(f)$ yy-quadrupole.

$\left(f=f^{*} h^{*} / u_{w}^{*}\right)$ as $R e_{w}$ increases. Above $f=1\left(R e_{w}=3400\right)$ or $f=0.4\left(R e_{w}=1300\right)$, the spectra run into numerical noise. The reason for using the lower Reynolds number in figures $19-21$ is the higher quality of the spectral statistics: because the $R e_{w}=3400$ case was expensive to compute, compromises had to be made with respect to box size and run time.

\subsection{Spectrum of radiated acoustic pressure in Poisenille flow}

In this section we show that the mean-square pressure at a point in the channel, produced by summation of contributions radiated from the entire flow field, has a spectrum that is independent of $M$ in the low- $M$ limit. This radiated contribution to the pressure spectrum is compared with the local spectrum obtained from the incompressible DNS, and found to be negligible except at low frequencies.

In the low- $M$ limit, the radiated plane-wave pressure in the channel is dominated by the wall-shear dipole term of (59). Substitution in (43) and integration over the 

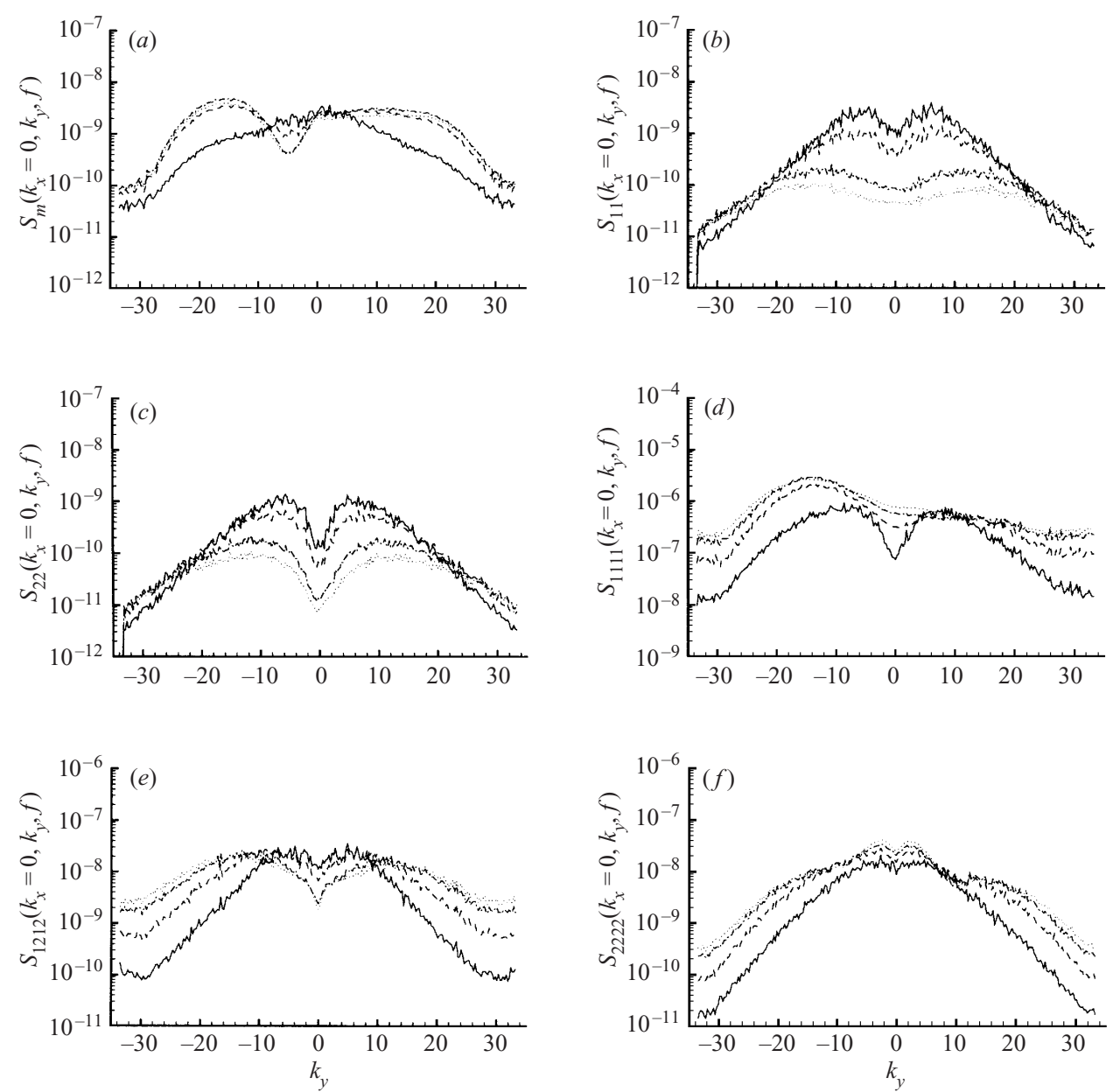

FIGURE 20. As figure 19 but for spanwise wavenumber $k_{y}$ with $k_{x}=0 . R_{w}=1300$.

entire channel gives

$$
\begin{aligned}
S_{p, \text { rad }}(f) & =\int_{0}^{2 \pi} \int_{0}^{\infty} S_{p}(R, \phi, f) \mathrm{e}^{-2 \alpha R} R \mathrm{~d} R \mathrm{~d} \phi \\
& \simeq \frac{\pi}{8} \frac{M}{f \alpha(f)}\left\{S_{11}(\mathbf{0}, f)+S_{22}(\mathbf{0}, f)\right\},
\end{aligned}
$$

where $R=|\boldsymbol{x}|, \phi$ is the azimuth angle and $\alpha(f)$ is the attenuation coefficient of the lowest mode in the channel; all quantities are scaled on the friction velocity and the channel half-width. The radiated acoustic pressure given by (67) is a two-dimensional diffuse field, whose strength is controlled by $\alpha$; in the absence of attenuation, the pressure would be infinite.

Asymptotic estimates of $\alpha(f)$, valid at low and high frequencies, have been presented in $\S 3.1$ based on the work of Howe (1995). For the purpose of estimating $S_{p \text {,rad }}$ from (67), we use the simple interpolation formula

$$
\alpha(f) \approx \alpha_{e}+\alpha_{0},
$$

where $\alpha_{e}$ is the low-frequency asymptote given by (24), and $\alpha_{0}$ is the flow-independent 

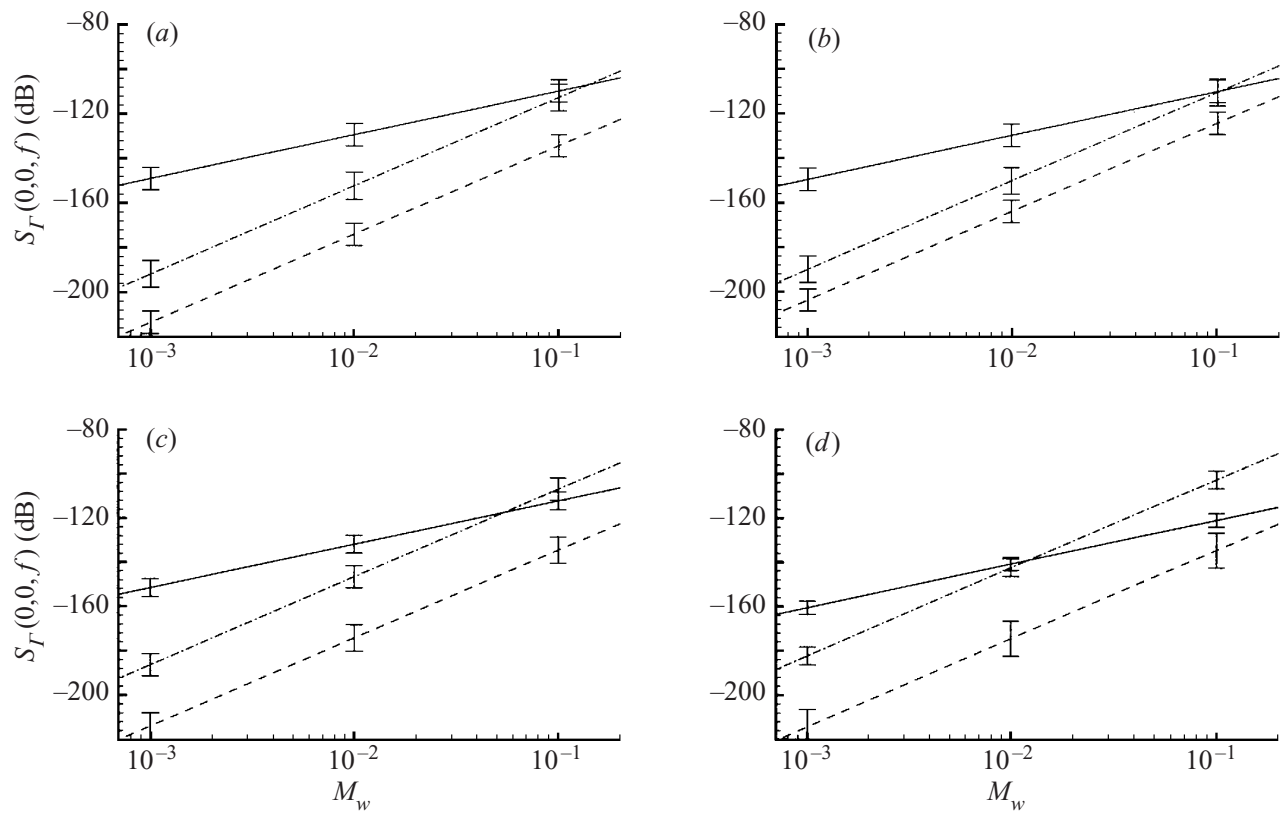

FiguRE 21. Contribution of different source terms to far-field sound radiation intensity of plane Couette flow in the streamwise direction. Error bars are estimated from the uncertainty of spectra near $(0,0)$ wavenumbers. Dashed line: monopole; solid line: $x$-dipole; dash-dotted line: $x x$-quadrupole. $R e_{w}=1300$. (a) $f=0.05$, (b) $f=0.1,(c) f=0.2$, (d) $f=0.4$.

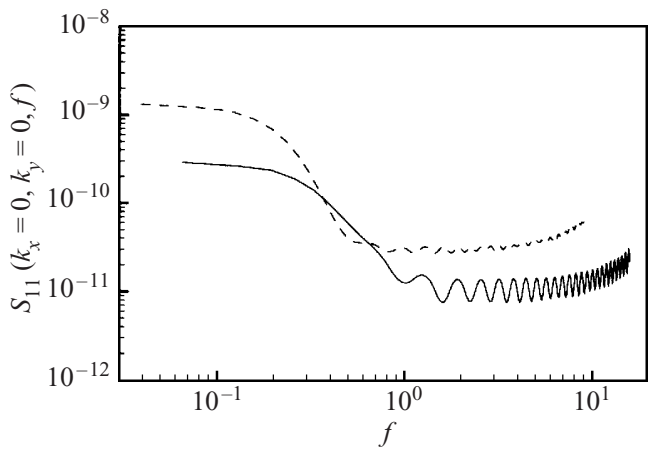

FIGURE 22. Frequency spectrum of the $x$-dipole source term $S_{11}$, scaled on wall velocity, at two different Reynolds numbers in plane Couette flow. $R e_{w}=3400$ (solid line), 1300 (dashed line). Compare figure 18 .

high-frequency asymptote given by (25). $\dagger$ Note that both $\alpha_{e}$ and $\alpha_{0}$ contain a factor $M_{\tau}$; substitution of (68) in (67) therefore leads to a radiated pressure spectrum that is independent of Mach number.

Figure 23 compares pressure spectra in the channel due to acoustic radiation with wall pressure spectra from incompressible DNS, for three different Reynolds numbers. At the lowest frequencies, the acoustic contribution approaches the local 'incompressible' pressure, with the closest approach occurring at $R e_{\tau}=135$. At higher

$\dagger$ Howe (1995) has developed a more sophisticated formula that shows good agreement with available measurements; his formula and (68) differ by at most a factor of 1.4, principally in the transition region between the low- and high-frequency asymptotes. 


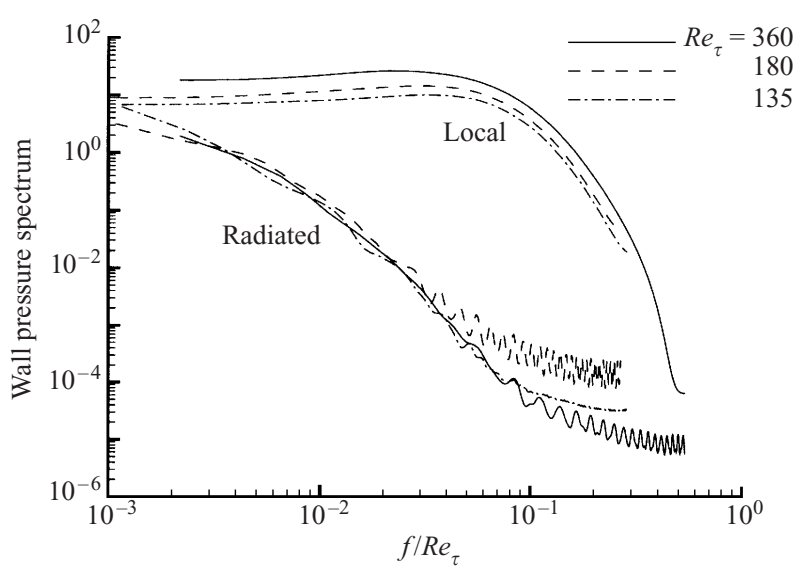

FIGURE 23. Normalized wall pressure spectra for turbulent Poiseuille flow in an infinite plane channel, simulated at three different Reynolds numbers. The upper curves are local pressure spectra obtained directly from incompressible DNS. The lower curves are asymptotic $(M \rightarrow 0)$ predictions of the radiated pressure at a point in the channel, estimated from equation (67). The ordinate is $S_{p}(f) R e_{\tau}$ (wall-variable scaling).

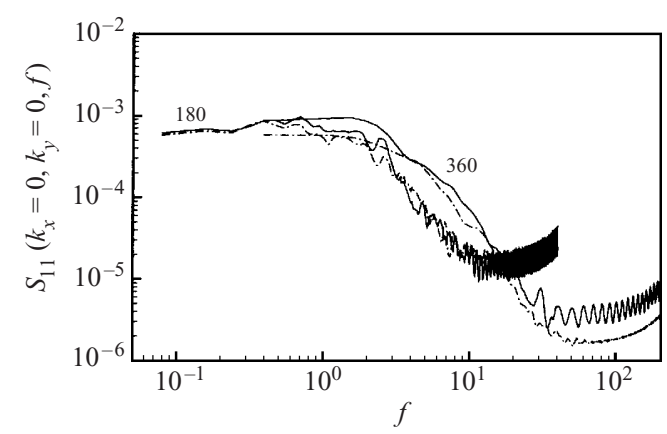

FIGURE 24. Box size comparison at zero wavenumber, for $x$-dipole source term $S_{11}$ in plane Poiseuille flow at two Reynolds numbers $\left(R e_{\tau}=360,180\right)$. Solid lines: larger box; dash-dotted lines: small box. Compare figure 18 .

frequencies, however, the acoustic contribution is several decades lower. It would be interesting to study the effect of $M_{\tau}$ on the turbulent wall pressure numerically, by running a series of compressible Poiseuille flow simulations, in order to examine whether compressibility does influence the pressure spectrum significantly at the low end as figure 23 suggests.

\subsection{Computational convergence}

The results shown above have been tested for convergence with respect to box size $\left(L_{x}, L_{y}\right)$ and record length $(T)$. Typical results for plane Poiseuille flow are discussed below. Box-size convergence for Couette flow has not been conclusively established; the one comparison available (at $R e_{w}=1300$ ) is with a box of only $(1 / 4 \times 1 / 2)$ the final dimensions (streamwise $\times$ spanwise), and a plot similar to figure 24 shows differences of order $5 \mathrm{~dB}$.

Figures 24 and 25 illustrate the effect of halving the box size in the streamwise and spanwise directions, at two values of $R e_{\tau}$, with the same temporal and spatial resolution as before. Results for the frequency dependence of the streamwise dipole 


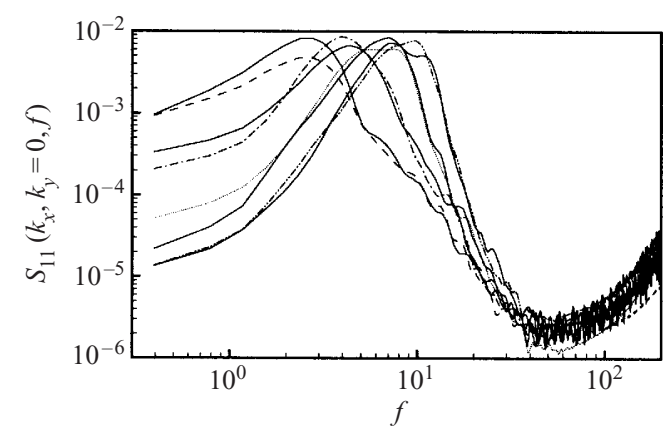

FIGURE 25. Box size comparison as in figure $24\left(R e_{\tau}=360\right)$, but for finite streamwise wavenumbers. Solid lines: results from larger box. For small box, dashed line: $k_{x}=1.05$; dash-dotted line: $k_{x}=2.09$; dotted line: $k_{x}=3.14$; dash-double-dotted line: $k_{x}=4.19$.

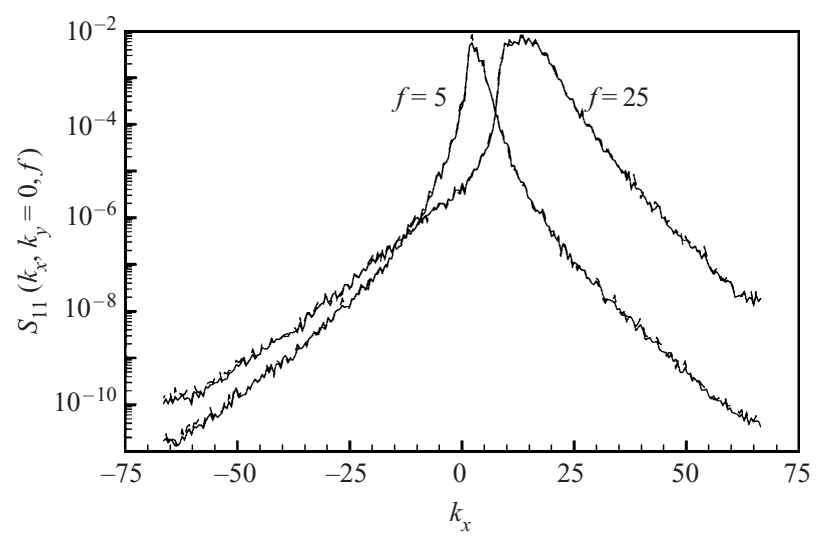

FigurE 26. Record length comparison at scaled frequency $f=5$ and $f=25$, for $x$-dipole source term $S_{11}$ in plane Poiseuille flow $\left(R e_{\tau}=360\right)$. Solid lines: $T=20$; dashed lines: $T=10$.

source term are compared for different streamwise wavenumbers, with spanwise wavenumber set to zero. It can be seen that the spectra agree well from $f=5$ up to the limit set by noise, at all wavenumbers. For low frequencies $(f<5)$, definite box-size convergence of the $R e_{\tau}=360$ simulation at low streamwise wavenumbers (of order $k_{x} \approx 3$ or less) seems to require a larger box than we have used.

The influence of record length has been checked for Poiseuille flow by comparing the spectra computed from different record lengths. Results from $T=20$, which is the standard record length used for all results presented so far, and $T=10$ collapse well throughout the frequency range of the present study ( $f=0.6$ to 25 ), as illustrated in figure 26 for $f=5$ and 25. This shows our results are not significantly influenced by the finite record length of the simulation.

\section{Discussion and conclusions}

Sound radiation from turbulence at low Mach number has been estimated numerically from first principles, for two well-defined flows in a plane channel. The method combines direct numerical simulations of turbulence, based on the incompressible Navier-Stokes equations, with a modified version of Lighthill's (1952) analogy in which the usual Reynolds-stress quadrupoles are supplemented by order- $M^{2}$ monopoles, equation (30), and order-1 surface dipoles, equation (29). The former arise 
from unsteady dissipation, and the latter from unsteady wall shear stresses. For both Poiseuille and Couette flow, it has been possible to obtain robust spectral estimates of all three source terms, and hence their relative contributions to the far-field intensity spectrum.

The incompressible DNS results (see also Hu et al. 2002) support Chase's (1991) conjecture that the wavenumber-frequency spectrum of wall shear-stress fluctuations, under an incompressible turbulent boundary layer, has a finite spectral density in the low-wavenumber limit. The same applies to the sum of the shear stresses on opposite walls of the channel, implying that the surface dipole distribution in the Lighthill analogy has a finite spectral density at zero wavenumber. It is argued (see below) that these low-wavenumber shear stresses exist independently of any acoustic response of the channel associated with compressibility.

It follows that the far-field radiation from turbulent channel flow is dipoledominated, in the low-Mach-number limit. The sound power per unit channel area varies asymptotically as $M^{2}$, rather than $M^{4}$ as would be the case for Reynolds stress radiation, with the latter contribution beginning to take over at low frequencies at a Mach number of order $M_{\max }=0.1$. The unsteady-dissipation contribution is generally small compared with that of the Reynolds-stress quadrupole components at high frequencies (figures 16, 17). However, for Poiseuille flow at low frequencies $(f<1$, figure 16), the two contributions in the spanwise radiation direction are within a factor of 2 at $R e_{\tau}=360$ (based on $\lambda=0.4$ ); and as the Reynolds number is reduced, the monopole contribution becomes dominant in this low-frequency region (figure 17).

A similar conclusion with regard to dipole dominance in wall-bounded flow at low Mach numbers was reached by Wang, Lele \& Moin (1996), based on incompressible DNS of an unstable wave packet undergoing transition in a flat-plate laminar boundary layer. The instantaneous shear force at the wall was integrated over a rectangular region under the transition spot, of extent $140 \delta^{*}$ (streamwise) by $25.95 \delta^{*}$ (spanwise), where $\delta^{*}$ is the displacement thickness of the inflow boundary layer. They found that 'If ... the wall shear stress is considered a generation term dominated by the hydrodynamic (non-acoustic) motion near the wall [then] at the late transition stage, the surface sound due to viscous stress fluctuations dominates the far field for a low-Mach-number flow....' Our findings from the channel flow study further support the view that incompressible-flow wall-stress fluctuations act as dipole sources of sound at low Mach number.

The process of sound generation in a turbulent boundary layer by unsteady wallshear stresses can be understood in terms of linear mode conversion at a solid boundary. Whereas small-amplitude perturbations of vorticity, entropy and pressure in a viscous fluid propagate independently in the absence of boundaries, coupling between these three disturbance modes can arise either from the boundary conditions or from bilateral interaction (Chu \& Kovásznay 1958). For our purpose, the relevant coupling mechanism is the conversion of an incident vorticity wave to an outgoing pressure wave, via viscous action at a plane boundary.

This mechanism for sound production, first proposed in the present context by Herbert, Leehey \& Haj-Hariri (1999), is familiar in elastodynamics as mode conversion from shear to dilatational waves. For vorticity waves in a viscous non-conducting fluid, it yields predictions that are identical to the Lighthill analogy in the limit $f M^{2} / R e \rightarrow 0$, both for a half-space and for a plane channel. According to this interpretation, the incident vorticity responsible for driving the process would be silent in the absence of the wall, despite its wavenumber parallel to the wall being smaller than the acoustic wavenumber $k_{0}$. The vorticity is scattered into sound via 
viscous stresses at the boundary, in a manner analogous to the scattering of vorticity at a trailing edge or a surface roughness element.

Importantly for the Lighthill analogy formulation, the linear model above reveals no significant back-reaction of the sound field on the wall-shear stress generated by an incident vorticity wave. It therefore appears justified to use wall-shear stresses generated by an incompressible simulation as inputs to the acoustic calculation of $\S 6$. Confirmation of the acoustic predictions made in that section must, nevertheless, eventually be sought via a fully compressible simulation of turbulent channel flow.

This study was supported by EPSRC under Grant GR/M38865, and the Cray T3E time was provided by EPSRC Grant GR/M08424. The authors thank Ian Castro and Sergei Chernyshenko for useful discussions.

\section{Appendix A. Scaling of density fluctuations in low-Mach-number turbulent flow}

\section{A.1. Non-conducting fluid}

In a non-reacting fluid of uniform composition, the density, $\rho$, can be expressed as a function of pressure, $P$, and specific entropy, $s$. Perturbations in density, relative to a reference state denoted by subscript 0 , are related to $P-P_{0}$ and $s-s_{0}$ by

$$
\rho-\rho_{0}=\frac{1}{c_{0}^{2}}\left(P-P_{0}\right)-\left(\frac{\rho \alpha T}{C_{p}}\right)_{0}\left(s-s_{0}\right)+(\text { quadratic terms }) ;
$$

here $C_{p}$ is the constant-pressure specific heat, $T$ is the absolute temperature, and $\alpha$ is the thermal expansivity of the fluid. (The variables are all dimensional, with the * superscript omitted.)

Suppose a turbulent shear layer is formed by the mixing of two uniform streams, having the same properties $\left(\rho_{0}, P_{0}, s_{0}\right)$ but different velocities. At low Mach number, the perturbations $\left(P-P_{0}, s-s_{0}\right)$ scale as follows, in terms of suitable velocity and length scales $\left(U_{\text {ref }}, L_{\text {ref }}\right)$ :

$$
P-P_{0} \sim \rho_{0} U_{\text {ref }}^{2}, \quad s-s_{0} \sim \frac{\Phi}{\rho_{0} T_{0}} \frac{L_{\text {ref }}}{U_{\text {ref }}} .
$$

Here $\Phi$ is the viscous dissipation rate per unit volume; it scales as

$$
\Phi \sim \rho_{0} \frac{U_{\mathrm{ref}}^{3}}{L_{\mathrm{ref}}},
$$

in turbulent flows at high Reynolds number. Note that the second of equations (A 2) follows from the entropy equation for a viscous non-conducting fluid (see Appendix B).

Substituting (A 2) and (A 3) in (A 1) gives

$$
\rho-\rho_{0} \sim\left(\rho_{0} M^{2}, \rho_{0} \lambda M^{2}\right)
$$

for the scaling of density fluctuations in adiabatic turbulent flows; here $M=U_{\text {ref }} / c_{0}$, and $\lambda=\alpha c^{2} / C_{p}$. The first term on the right is associated with fluctuations in pressure, and the second term with fluctuations in entropy; however, both scale as $M^{2}$.

A similar argument may be applied to the isentropic compressibility, $K=\rho^{-1} c^{-2}$, to yield the scaling relation

$$
K-K_{0} \sim K_{0} M^{2} .
$$




\section{A.2. Influence of thermal conduction}

In a turbulent shear layer formed by two streams of the same temperature (isothermal mixing),

$$
T-T_{0} \sim T_{0} M^{2},
$$

according to the argument of the previous section. Estimation of the thermal conduction term $\left(-\partial q_{i} / \partial x_{i}\right)$ in the entropy equation of Appendix B, using (A 6), shows it to be of order $M^{2}$ smaller than the viscous term under these conditions. Equations (A 4) and (A 5) therefore remain valid for a thermally conducting fluid.

\section{Appendix B. Relation between $R$ and the viscous dissipation function}

The excess dilatation rate defined in equation (10) of the main text may be rewritten in dimensional variables, using the continuity equation, as

$$
R=-\frac{1}{\rho} \frac{\mathrm{D} \rho}{\mathrm{D} t}+\frac{1}{\rho c^{2}} \frac{\mathrm{D} P}{\mathrm{D} t} .
$$

(All variables are dimensional, as in Appendix A, with the * superscript omitted for convenience.) In a simple non-reacting fluid of uniform composition, it follows that $R$ is related to $s$ (specific entropy) by

$$
R=\frac{\alpha T}{C_{p}} \frac{\mathrm{D} s}{\mathrm{D} t},
$$

where $\alpha$ is the volume thermal expansivity, and $C_{p}$ is the constant-pressure specific heat.

The material derivative $\mathrm{Ds} / \mathrm{D} t$ is related through the energy equation to the viscous stress (components $\tau_{i j}$ ) and heat flux (components $q_{i}$ ) as follows:

$$
\rho T \frac{\mathrm{D} s}{\mathrm{D} t}=\frac{\partial u_{i}}{\partial x_{j}} \tau_{i j}-\frac{\partial q_{i}}{\partial x_{i}} .
$$

The first term on the right is the viscous dissipation function $\Phi$; the second term is zero, in a non-conducting fluid with no radiative heat transfer.

From (B 2) and (B 3), for the restricted class of flows defined above,

$$
R=\frac{\alpha}{\rho C_{p}} \Phi \text { (adiabatic, non-reacting, uniform composition). }
$$

We now introduce the non-dimensional Grüneisen parameter $\lambda$, defined by

$$
\lambda=\frac{\rho}{T}\left(\frac{\partial T}{\partial \rho}\right)_{s}=\frac{\alpha c^{2}}{C_{p}} .
$$

Combining (B 4) and (B 5) and reverting to scaled variables - as defined in $§ 2.1$ - gives the final result

$$
R=\lambda M^{2} \Phi+O\left(M^{4}\right) .
$$

Here Appendix A has been used to write $c-c_{0} \sim c_{0} M^{2}$ and $\rho-\rho_{0} \sim \rho_{0} M^{2}$. Note that introducing a finite thermal conductivity leads to an $O\left(M^{4}\right)$ correction term in (B 6), for isothermal mixing; see $\S$ A.2 of Appendix A. 
ARP, V. 1975 Thermodynamics of single-phase one-dimensional fluid flow. Cryogenics 15, 285-289.

Canuto, C., Hussaini, M. Y., Quarteroni, A. \& Zang, T. A. 1987 Spectral Methods in Fluid Dynamics. Springer.

Chase, D. M. 1991 Fluctuations in wall-shear stress and pressure at low streamwise wavenumbers in turbulent boundary-layer flow. J. Fluid Mech. 225, 545-555.

Chu, B.-T. \& Kovásznay, L. S. G. 1958 Non-linear interactions in a viscous heat-conducting compressible gas. J. Fluid Mech. 3, 494-514.

Davies, H. G. \& Ffowcs Williams, J. E. 1968 Aerodynamic sound generation in a pipe. J. Fluid Mech. 32, 765-778.

Ffowcs Williams, J. E. \& Hawkings, D. L. 1968 Shallow water wave generation by unsteady flow. J. Fluid Mech. 31, 779-788.

Ffowcs Williams, J. E. \& Hawkings, D. L. 1969 Sound generation by turbulence and surfaces in arbitrary motion. Phil. Trans. R. Soc. Lond. A 264, 321-342.

Herbert, K., Leehey, P. \& Haj-Hariri, H. 1999 On the Mach- and Reynolds-number dependence of the flat-plate turbulent boundary layer wall-pressure spectrum. Theor. Comput. Fluid Dyn. 13, 33-56.

Howe, M. S. 1995 The damping of sound by wall turbulent shear layers. J. Acoust. Soc. Am. 98, $1723-1730$.

Hu, Z. W., Morfey, C. L. \& Sandham, N. D. 2002 Aeroacoustics of wall-bounded turbulent flows. AIAA J. 40, 465-473.

Hu, Z. W. \& SAndham, N. D. 2001 Large-domain simulations of plane Couette and Poiseuille flow. In Proc. 2nd Intl Symp. on Turbulence Shear Flow Phenomena (ed. E. Lindborg et al.), pp. 377-382. KTH, Stockholm.

IAFRATI, A. \& RiCCARDI, G. 1996 A numerical evaluation of viscous effects on vortex induced noise. J. Sound Vib. 196, 129-146.

Kambe, T. 1984 Influence of viscosity on aerodynamic sound emission in free space. J. Sound Vib. 95, 351-360.

Kambe, T. \& Minota, T. 1983 Acoustic wave radiated by head-on collision of two vortex rings. Proc. R. Soc. Lond. A 386, 277-308.

Kim, J., Moin, P. \& Moser, R. 1987 Turbulence statistics in fully developed channel flow at low Reynolds number. J. Fluid Mech. 177, 133-166.

Kleiser, L. \& Schumann, U. 1980 Treatment of incompressibility and boundary layer conditions in 3D numerical spectral simulations of plane channel flows. In Proc. 3rd GAMM Conf. on Numerical Methods in Fluid Mechanics (ed. E. H. Hirschel), pp. 165-173. Vieweg.

Komminaho, J., Lundbladh, A. \& Johansson, A. 1996 Very large structure in plane turbulent Couette flow. J. Fluid Mech. 320, 259-285.

Kristoffersen, R., Bech, K. H. \& Andersson, H. I. 1993 Numerical study of turbulent plane Couette flow at low Reynolds number. Appli. Sci. Res. 51, 337-343 (referred to herein as KBA).

Lighthill, M. J. 1952 On sound generated aerodynamically: I. General theory. Proc. R. Soc. Lond. A 211, 564-587.

Lighthill, M. J. 1954 On sound generated aerodynamically: II. Turbulence as a source of sound. Proc. R. Soc. Lond. A 222, 1-32.

Lighthill, M. J. 1978 Waves in Fluids. Cambridge University Press.

Mansour, N. N., Kim, J. \& MoIn, P. 1988 Reynolds-stress and dissipation-rate budgets in a turbulent channel flow. J. Fluid Mech. 194, 15-44.

Mitchell, B. E., Lele, S. K. \& Moin, P. 1995 Direct computation of the sound from a compressible co-rotating vortex pair. J. Fluid Mech. 285, 181-202.

Morfey, C. L. 1973 Amplification of aerodynamic noise by convected flow inhomogeneities. J. Sound Vib. 31, 391-397.

Morfey, C. L. 1976 Sound radiation due to unsteady dissipation in turbulent flows. J. Sound Vib. 48, 95-111.

Morfey, C. L. 1999 Aeroacoustics of turbulent flows with dissipation. In Proc. 6th Intl Congress on Sound and Vibration (ed. F. Jacobsen), vol. 7, pp. 3631-3642. DTU, Copenhagen.

Morse, P. M. \& Ingard, K. U. 1986 Theoretical Acoustics 2nd edn. Princeton University Press. 
Moser, R. D., Kim, J. \& Mansour, N. N. 1999 Direct numerical simulation of turbulent channel flow up to $R e_{\tau}=590$. Phys. Fluids 11, 943-945 (referred to herein as MKM).

Obermeier, F. 1967 Berechnung aerodynamisch erzeugter Schallfelder mittels der Methode der 'Matched Asymptotic Expansions'. Acustica 18, 238-240.

Obermeier, F. 1985 Aerodynamic sound generation caused by viscous processes. J. Sound Vib. 99, $111-120$.

Obermeier, F. \& Möhring, W. 1984 Aerodynamic sound generation by turbulent boundary layer flows along solid and compliant walls. Z. Flugwiss. Weltraumforsch. 8, 181-192.

Pagneux, V. \& Froehlich, B. 2001 Influence of low Mach number shear flow on acoustic propagation in ducts. J. Sound Vib. 246, 137-155.

Peters, M. C. A. M., Hirschberg, A., Reijnen, A. J. \& Wijnands, A. P. J. 1993 Damping and reflection coefficient measurements for an open pipe at low Mach and low Helmholtz numbers. J. Fluid Mech. 256, 499-534.

Pierce, A. D. 1989 Acoustics: An Introduction to its Physical Principles and Applications (1st edn of 1981, reprinted with corrections). Acoustical Society of America, New York.

Powell, A. 1960 Aerodynamic noise and the plane boundary. J. Acoust. Soc. Am. 32, 982-990.

SANDHAM, N. D. \& HowARD, R. J. A. 1998 Direct simulation of turbulence using massively parallel computers. In Parallel Computational Fluid Dynamics (ed. D. R. Emerson et al.), pp. 23-32. Elsevier.

Tillmark, N. \& Alfredsson, P. H. 1993 Turbulence in plane Couette flow. Appl. Sci. Res. 51, $237-241$

WANG, M., Lele, S. K. \& Moin, P. 1996 Sound radiation during local laminar breakdown in a low-Mach-number boundary layer. J. Fluid Mech. 319, 197-218. 